\title{
Causes of Robust Seasonal Land Precipitation Changes*
}

\author{
Debbie Polson and Gabriele C. Hegerl \\ School of GeoSciences, University of Edinburgh, Edinburgh, United Kingdom \\ XUEBIN ZHANG \\ Climate Research Division, Environment Canada, Toronto, Ontario, Canada \\ TIMOTHY J. OSBORN \\ Climatic Research Unit, School of Environmental Sciences, University of East Anglia, Norwich, \\ Norfolk, United Kingdom
}

(Manuscript received 23 July 2012, in final form 30 January 2013)

\begin{abstract}
Historical simulations from phase 5 of the Coupled Model Intercomparison Project (CMIP5) archive are used to calculate the zonal-mean change in seasonal land precipitation for the second half of the twentieth century in response to a range of external forcings, including anthropogenic and natural forcings combined (ALL), greenhouse gas forcing, anthropogenic aerosol forcing, anthropogenic forcings combined, and natural forcing. These simulated patterns of change are used as fingerprints in a detection and attribution study applied to four different gridded observational datasets of global land precipitation from 1951 to 2005 . There are large differences in the spatial and temporal coverage in the observational datasets. Yet despite these differences, the zonal-mean patterns of change are mostly consistent except at latitudes where spatial coverage is limited. The results show some differences between datasets, but the influence of external forcings is robustly detected in March-May, December-February, and for annual changes for the three datasets more suitable for studying changes. For June-August and September-November, external forcing is only detected for the dataset that includes only long-term stations. Fingerprints for combinations of forcings that include the effect of greenhouse gases are similarly detectable to those for ALL forcings, suggesting that greenhouse gas influence drives the detectable features of the ALL forcing fingerprint. Fingerprints of only natural or only anthropogenic aerosol forcing are not detected. This, together with two-fingerprint results, suggests that at least some of the detected change in zonal land precipitation can be attributed to human influences.
\end{abstract}

\section{Introduction}

Increased temperatures over the twentieth century have led to an increase in the moisture content of the atmosphere (Santer et al. 2007; Willett et al. 2007), and changes to global (Zhang et al. 2007; Huffman et al. 2009; Smith et al. 2010) and regional precipitation

\footnotetext{
* Supplemental information related to this paper is available at the Journals Online website: http://dx.doi.org/10.1175/ JCLI-D-1200474.s1.

Corresponding author address: Debbie Polson, School of GeoSciences, Grant Institute, The University of Edinburgh, The King's Buildings, West Mains Road, Edinburgh EH9 3JW, United Kingdom.

E-mail: dpolson@staffmail.ed.ac.uk
}

patterns have been observed (e.g., Hoerling et al. 2006; Min et al. 2008; Kang et al. 2011) that follow expectations based on physics and modeling (Held and Soden 2006). Global and regional precipitation changes may be influenced by a complex range of factors including the direct response to greenhouse gas forcing and to the warming of the atmosphere (Allen and Ingram 2002; Lambert and Allen 2009), changes in atmospheric circulation (e.g., Ineson and Scaife 2009; Kenyon and Hegerl 2010), sea surface temperature changes (e.g., Hoerling et al. 2012; Yoshioka et al. 2007; Lyon and DeWitt 2012; Hoerling et al. 2012), and regional changes in vegetation (e.g., Wang et al. 2004) and stratospheric aerosols (e.g., Gillett et al. 2004).

One of the predicted responses of the hydrological cycle to increasing greenhouse gas concentrations is the intensification of the water cycle (Allen and Ingram 
2002; Held and Soden 2006; Wentz et al. 2007; Chou et al. 2009; Seager et al. 2010). As the atmosphere warms, water vapor increases in line with the ClausiusClapeyron relation and as the horizontal transport of water vapor increases, the existing pattern of precipitation minus evaporation $(P-E)$ is enhanced. Thus, areas that export moisture get drier and areas that import water get wetter (Held and Soden 2006). However, the increase in global precipitation is constrained by tropospheric radiative cooling with precipitation increasing at a slower rate than column water vapor (Allen and Ingram 2002; Lambert and Allen 2009). There must therefore be a corresponding decrease in the convective mass flux, implying weakening atmospheric circulation in the tropics, where most moist convection occurs (Vecchi et al. 2006; Lu et al. 2007). Changes in the mean circulation in response to warming will also affect precipitation. In particular, warming is expected to lead to a poleward expansion of the Hadley cells with an associated poleward expansion of the subtropical dry regions and poleward shift of the midlatitude storm tracks (Yin 2005; Lorenz and DeWeaver 2007; Seidel et al. 2008; Lu et al. 2007; Seager et al. 2010; Scheff and Frierson 2012), though the exact mechanisms are still to be understood.

Held and Soden (2006) show that the zonal-mean changes in $P-E$ are robust in response to warming associated with greenhouse gas forcing and that the structure of these changes is dominated by changes in precipitation. Consequently, Zhang et al. (2007) and Noake et al. (2012) used zonal means to show the global patterns of land precipitation change, for which longterm records of precipitation are available, and to derive fingerprints of forcing that can be used for detection and attribution. This enhancement of the $P-E$ pattern is less clear over land than oceans, where evaporation can be limited in arid regions. Model-simulated future precipitation change broadly follows a similar zonal pattern of moistening and drying over ocean as over land (Meehl et al. 2007), and significant changes in precipitation over land due to human influence are expected to have already occurred (Balan Sarojini et al. 2012).

The observed changes in precipitation are a combination of external forcing and internal variability (together with errors and biases arising from limitations of the observational system). Internal variations can cause regional changes in precipitation on multidecadal time scales (Dai 2013), making it difficult to distinguish the influence of external forcing. The spatial scale of these decadal features tends to be regional (Cayan et al. 1998), so by averaging over large areas, such as zonal means, the influence of internal variability on the observed precipitation changes can be reduced. The internal variations in the model simulations will be unique to each simulation.
By averaging over many simulations, the influence of internal variability is reduced in the patterns of precipitation change (Zhang et al. 2007; Knutti et al. 2010).

Fingerprint detection and attribution methods (Allen and Stott 2003) have been used to attribute observed changes in precipitation over the latter twentieth century to anthropogenic forcing for annual global zonal land precipitation (Zhang et al. 2007) and for the Arctic (Min et al. 2008) and to attribute changes in seasonal global zonal land precipitation to external forcing (Noake et al. 2012) using phase 3 of the Coupled Model Intercomparison Project (CMIP3) archive. Estimates of the mean zonal changes in annual precipitation found the multimodel mean and individual model simulations from the CMIP3 archive tended to underestimate the magnitude of the observed precipitation change (Zhang et al. 2007). However, using seasonal zonal-mean land changes expressed as percentages from three different datasets of near-global coverage, Noake et al. (2012) found that observed precipitation changes were significantly underestimated by the models for all the datasets only during March-May (MAM). For other seasons, models and observations were consistent given data uncertainty and internal variability, though the dataset used in Zhang et al. (2007) also showed the models underestimating the changes in December-February (DJF) and annual precipitation. The precipitation changes for three observational datasets and models were found to be quite similar to each other where the observations and models were masked to include only grid boxes where gauge data were available. External forcing was detectable in DJF, MAM, and September-November (SON) for all observational datasets and in June-August (JJA) for one dataset at the 5\% level of significance; however, the authors did not separate the response of anthropogenic forcing from natural forcing and could not therefore demonstrate that anthropogenic forcing had contributed significantly to seasonal patterns of change as it had been shown to for annual changes (Zhang et al. 2007).

Here, we apply the methodology of Noake et al. (2012) to the models from the new phase 5 of the Coupled Model Intercomparison Project (CMIP5) archive (Taylor et al. 2012) and four observational datasets to compare the performance of the latest generation of models to that of the previous generation. In addition to applying total least squares detection to a fingerprint of all external forcings (ALL) ${ }^{1}$ forcings, the analysis is extended to

\footnotetext{
${ }^{1}$ Here, "ALL" external forcings refers to the full set of forcings prescribed in the CMIP5 protocol as implemented by each participating modeling center.
} 
include fingerprints of greenhouse gas-only (GHG) forcing, anthropogenic-only (ANT) forcing, natural-only (NAT) forcing, and anthropogenic aerosol-only (AA) forcing in order to separate the contribution of different forcings to the observed pattern of change.

The paper is organized as follows: section 2 briefly introduces the detection and attribution method and section 3 introduces the data. In section 4 , robust and less robust changes in zonal precipitation are discussed across observational data and model simulations. Section 5 gives detection and attribution results that are discussed in section 6 .

\section{Total least squares detection}

The goal of detection and attribution is to determine if observations show evidence of forced changes expected from physical reasoning (e.g., as captured in climate models). In climate change detection and attribution, $\mathbf{F}$ is an $l \times p$ matrix of fingerprints that define response patterns (rank- $l$ vectors, such as zonal-mean precipitation changes) to $p$ external climate forcings. It is scaled to the rank- $l$ vector of observations $\mathbf{y}$ to estimate the magnitude of each pattern in the observed climate, using total least squares (tls) regression (Allen and Stott 2003):

$$
\mathbf{y}=\left(\mathbf{F}+\boldsymbol{\varepsilon}_{\text {finger }}\right) \cdot \boldsymbol{\beta}+\boldsymbol{\varepsilon}_{\text {noise }},
$$

where $\boldsymbol{\beta}$ is a vector of scaling factors with $p$ entries for each forcing fingerprint, $\boldsymbol{\varepsilon}_{\text {noise }}$ is the residual associated with internal climate variability, and $\boldsymbol{\varepsilon}_{\text {finger }}$ is the variability superimposed on the fingerprint. As signal-tonoise ratio is low for precipitation, $\boldsymbol{\varepsilon}_{\text {finger }}$ will be nonnegligible even for a multimodel mean. If the scaling factor exceeds zero at a particular significance level, then the fingerprint response pattern is detected in the observations (see Hegerl et al. 2007).

Detailed theoretical discussion of the tls method can be found in Allen and Stott (2003); here, we describe the practical implementation. We define the $l \times p^{\prime}$ matrix $\mathbf{Z} \equiv[\mathbf{F}, \mathbf{y}]$, where $p^{\prime}=p+1$, and take the singular value decomposition $\mathbf{Z}=\mathbf{U} \mathbf{\Lambda} \mathbf{V}^{\mathrm{T}}$. After sorting eigenvalues into descending order, the $p^{\prime}$ th element of the $p^{\prime}$ th column of $\mathbf{V}$, the $p^{\prime}$-rank vector $\tilde{\mathbf{v}}$ corresponds to the bestfit scaling parameter. The scaling factor for fingerprint $i$ is then $\boldsymbol{\beta}_{i}=-\tilde{\mathbf{v}}_{i} / \tilde{\mathbf{v}}_{p^{\prime}}$ (Allen and Stott 2003).

Internal climate variability leads to uncertainty in the fingerprint and observed response patterns and hence to uncertainty in the scaling factor. This uncertainty must be taken into account when applying detection and attribution. Only when the probability that $\beta$ is consistent with zero is small (e.g., $5 \%$ ) can it be stated with confidence that the fingerprint response pattern has been detected in the observations. The uncertainty in the scaling factor can be calculated by superimposing multiple samples of noise onto both the fingerprint and observations and recalculating the scaling factor. Each noise sample is a rank- $l$ vector, meaning that the variance does not have to be the same for all the elements (so the strength of internal variability can vary with latitude; see the supplemental material available at the Journals Online website: http://dx.doi.org/10.1175/ JCLI-D12-00474.s1) and that the covariance pattern of precipitation variability is preserved. Where $95 \%$ of the $\boldsymbol{\beta}$ values exceed zero, it is concluded that there is a significant $(p<5 \%)$ relationship between the observed and multimodel changes that cannot be explained by internal climate variability.

For the tls method, noise-reduced observation and model fingerprints are calculated using

$$
\tilde{\mathbf{Z}}=\mathbf{Z}-\mathbf{Z} \tilde{\mathbf{v}} \tilde{\mathbf{v}}^{\mathrm{T}}
$$

to produce a "best fit" of both. It is onto these that the sample of noise is added for evaluating $\boldsymbol{\beta}$. The regression residual $\boldsymbol{\varepsilon}_{\text {noise }}$ is compared to samples of model variability using the $F$ test of Allen and Tett (1999).

We experimented with using optimal fingerprints but found that the benefit was small (i.e., the results were similar) compared to the level of complexity introduced by having to truncate to a low-dimensional space, so the results shown here are for nonoptimized fingerprints (e.g., Zhang et al. 2007).

\section{Data: Observations and models}

Four gridded observational datasets for monthly precipitation were used in this analysis. The datasets are an updated version of data from Zhang et al. (2007): the Climate Research Unit (CRU) monthly precipitation dataset [updated from CRU TS3.1 of Harris et al. (2013)] and two datasets from the Global Precipitation Climatology Centre (GPCC), the Variability Analysis of Surface Climate Observations (VASClimO) dataset (Beck et al. 2005) and Full Reanalysis dataset (Schneider et al. 2013). While each gridded dataset is constructed independently, they use overlapping sets of the same raw station data. Although the stations do not provide complete spatial and temporal coverage, Wan et al. (2013) showed that they are likely to be sufficient for estimating the magnitude of changes and variability in regional land area mean precipitation.

The GPCC dataset is spatially interpolated covering the period 1901-2010. It is used with caution as inhomogeneities such as the variable number of stations per grid square over time can cause artifacts when calculating 
changes (Beck et al. 2005) but is included as it uses the largest number of stations of the four datasets considered here. The VASClimO dataset is a homogenized GPCC product adjusted to support climate variability and change analyses; however, data are only available until 2000 with an update in preparation (A. Becker 2012, personal communication). For completeness, both the datasets are included in the analysis; however, the results for the VASClimO may be more reliable because of the homogenization work. The VASClimO dataset consists of $2.5^{\circ} \times 2.5^{\circ}$ data from 1951 to 2000 and is based on quality-controlled and homogenized time series from 9343 stations. The data in Zhang et al. (2007) are on a $5^{\circ} \times 5^{\circ}$ grid based on long-term stations in the Global Historical Climatology Network (GHCN) monthly precipitation dataset (Vose et al. 1992). The authors selected stations with at least $25 \mathrm{yr}$ of data during 196190 and at least $5 \mathrm{yr}$ of data in every decade during 1950 99. The updated version covers the period 1900-2009 and replaces Canadian stations in the GHCN with homogeneity-adjusted Canadian data (Mekis and Vincent 2011). The CRU dataset, updated from CRU TS3.1, covers the period from 1901 to 2010 and uses station data collated from various sources (see Harris et al. 2013) similar to those used by GHCN. No homogenization or bias corrections are applied by CRU beyond those already implemented by the data sources. The station data are interpolated to provide precipitation estimates at each $0.5^{\circ} \times 0.5^{\circ}$ grid box. Here, grid box values that lie within $450 \mathrm{~km}$ of an observed monthly value are aggregated to a $5^{\circ} \times 5^{\circ}$ grid and only grid boxes containing stations in at least two of the aggregated $0.5^{\circ} \times 0.5^{\circ}$ grid boxes (or one if at least $50 \%$ of the $5^{\circ} \times 5^{\circ}$ grid box is ocean) are included in this analysis.

Together the four datasets cover a range of methods used to construct gridded observations. The Zhang dataset provides robust long-term homogenized records, but poor spatial coverage in some regions. While the GPCC includes both long- and short-term station data and extensive interpolation and provides complete spatial coverage over land, it introduces significant uncertainty in changes of precipitation over time that will be more affected by changing station coverage than the Zhang dataset. The VASClimO dataset addresses some of the homogeneity issues of the GPCC but still includes significant interpolation and infilling of grid boxes. The CRU dataset has been restricted, in this analysis, to only grid boxes where some station data exist and provide more spatial coverage than the Zhang dataset but without the same consistency in the station records over time.

All datasets are gridded to the same $5^{\circ} \times 5^{\circ}$ grid but are not masked to be spatially consistent with each other and therefore spatial coverage varies between datasets, as seen in Fig. 1. This illustrates the value of using multiple datasets-for example, the CRU dataset has poorer coverage than the Zhang dataset in Canada but better coverage across much of northern Asia. Temporal coverage also varies between datasets with data only available from 1951 to 2000 for the VASClimO data; therefore, the calculation of changes is limited to 1952-2000 for this dataset. For the CRU, Zhang, and GPCC datasets, changes were calculated for 1951-2005. Grid boxes were excluded where data were available in less than $95 \%$ of the years to minimize the impact of changes in the spatial coverage of the data over time.

The CMIP5 archive, accessed in December 2012, included 98 simulations from 33 models forced with anthropogenic and natural external forcing to derive a fingerprint of ALL-forced change, 45 simulations from 15 models for GHG-forced change, 38 simulations from 15 models for NAT-forced change, 18 simulations from 6 models for ANT-forced change, and 16 simulations from 7 models for AA-forced change. For each observation dataset, the simulations are transferred to the observational grid and masked to match the spatial and temporal data availability of that dataset on a gridbox basis. The models used in this analysis are listed in Table 1.

\section{Zonal patterns of precipitation change}

Zonal-mean change in precipitation is calculated by applying a linear least squares regression to precipitation averaged across each zonal band and is expressed as the percentage change in precipitation relative to the climatologically averaged precipitation of each $5^{\circ}$ zonal band between $45^{\circ} \mathrm{S}$ and $75^{\circ} \mathrm{N}$ giving spatial dimension $l=24$. Expressing changes as percentages rather than anomalies has been shown to improve agreement between models and between models and observations (Noake et al. 2012) and better represents the changes that may be small but important in relatively dry regions (e.g., Jones and Hulme 1996). For each 3-month season, the absolute precipitation in each $5^{\circ}$ latitude band is averaged and a linear least squares fit is applied over all the years to calculate the change in precipitation. This absolute change is then divided by the mean precipitation over the same period, spatial coverage, and latitude band, and is then converted to a percentage. This zonal percentage change is more robust than percentage change for individual grid boxes, as otherwise very high values can result for climatologically extremely dry grid boxes.

The simulated zonal-precipitation change pattern is used to derive the fingerprints of forcing where $\mathbf{F}$ is the multimodel-mean fingerprint from the ensembles of ALL-forced simulations, GHG-forced simulations, 


\section{CRU}
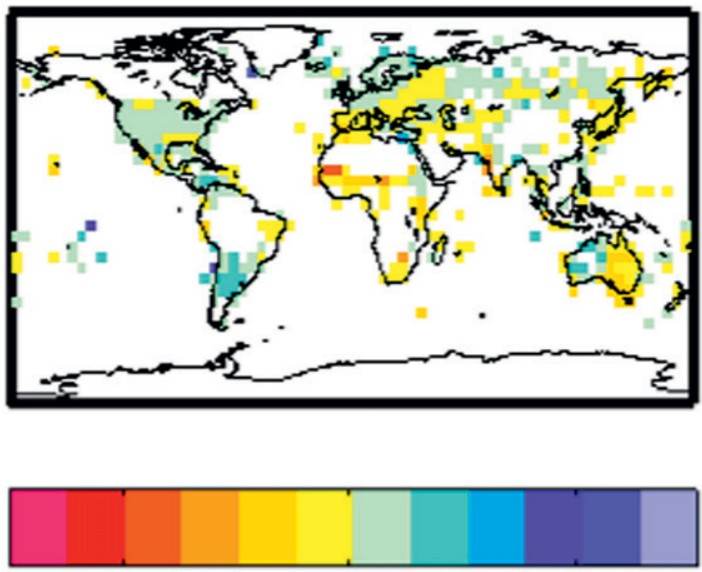

20

VASCLIMO
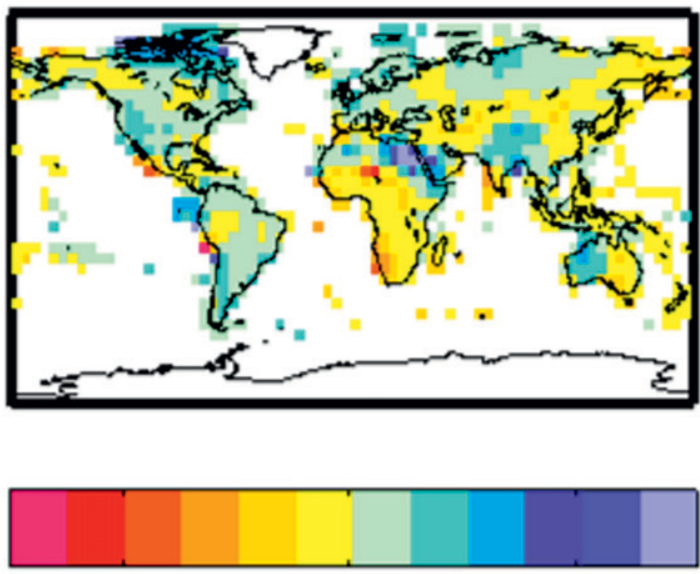

$-20$

20
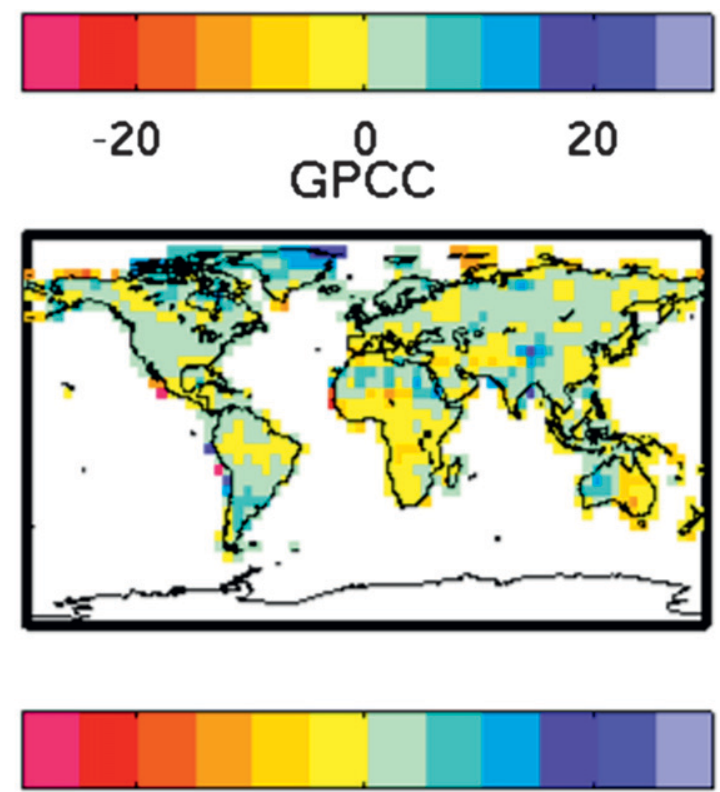

$-20$

20

20
ZHANG

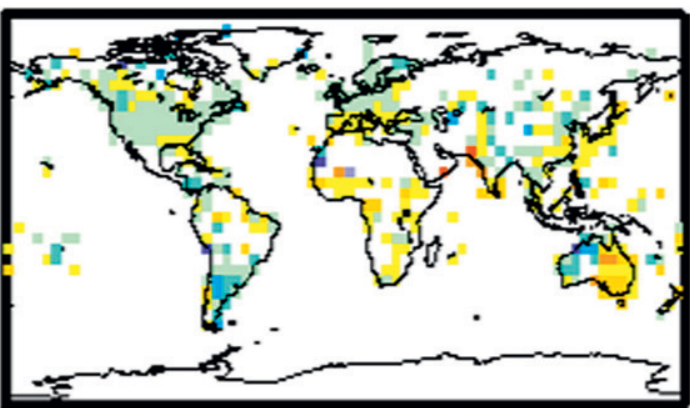

FIG. 1. Percentage change in precipitation per decade for the CRU (1951-2005), Zhang (1951-2005), VASClimO (1952-2000), and GPCC (1951-2005) datasets for MAM.

NAT-forced simulations, ANT-forced simulations, and AA-forced simulations. One-signal detection (i.e., $p=1$ ) is applied to the seasonal and annual ALL, GHG, NAT, ANT, and AA fingerprints to fit each fingerprint individually to the observed zonal-mean patterns of change $\mathbf{y}$ calculated from each observation dataset. A two-signal detection $(p=2)$ was also applied to annualchange pattern fingerprints (considering two combinations: first GHG and AA forcing and second ANT and NAT forcing) to determine if the observed change can be attributed to a combination of external forcings by distinguishing the role of individual forcings in observed precipitation patterns.

Samples of seasonal and annual zonal-mean precipitation changes associated with internal climate variability were derived by subtracting the multimodel mean from each individual simulation's zonal-mean pattern of change from the ALL forcing simulations and multiplying by $\sqrt{n / n-1}$, where $n$ is the number of simulations in the ensemble to avoid bias in the variance (Von Storch and Zwiers 2001). Similarly, the samples of noise on the fingerprint for each of the individual forcings were calculated by subtracting the multimodel mean from each individual simulation. To account for possible underestimation of the precipitation variability in some latitude bands by models [see Zhang et al. (2007) and Fig. S1 in the supplemental material], the scaling factors were also calculated using double the model variance for the samples of noise. 


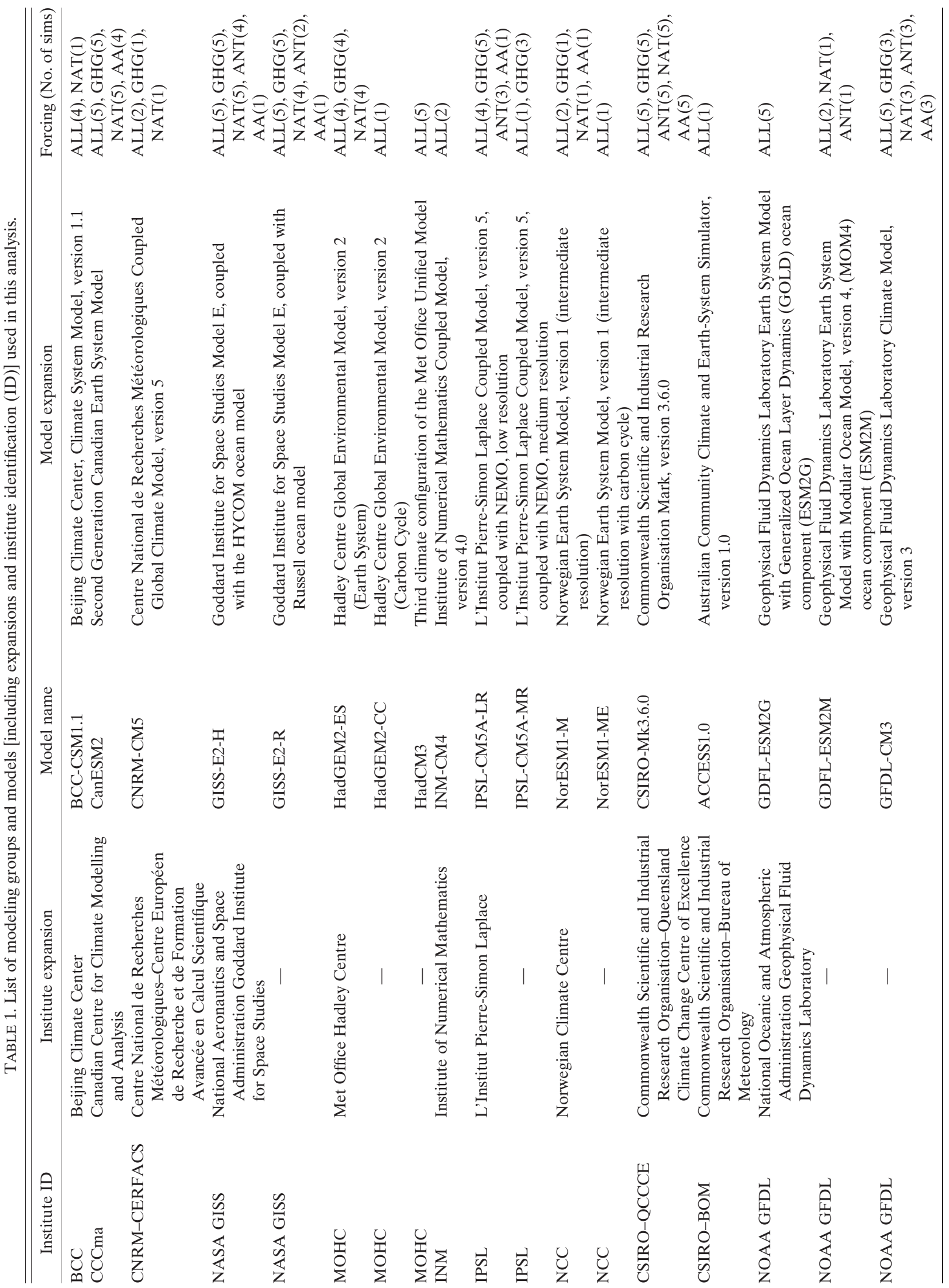




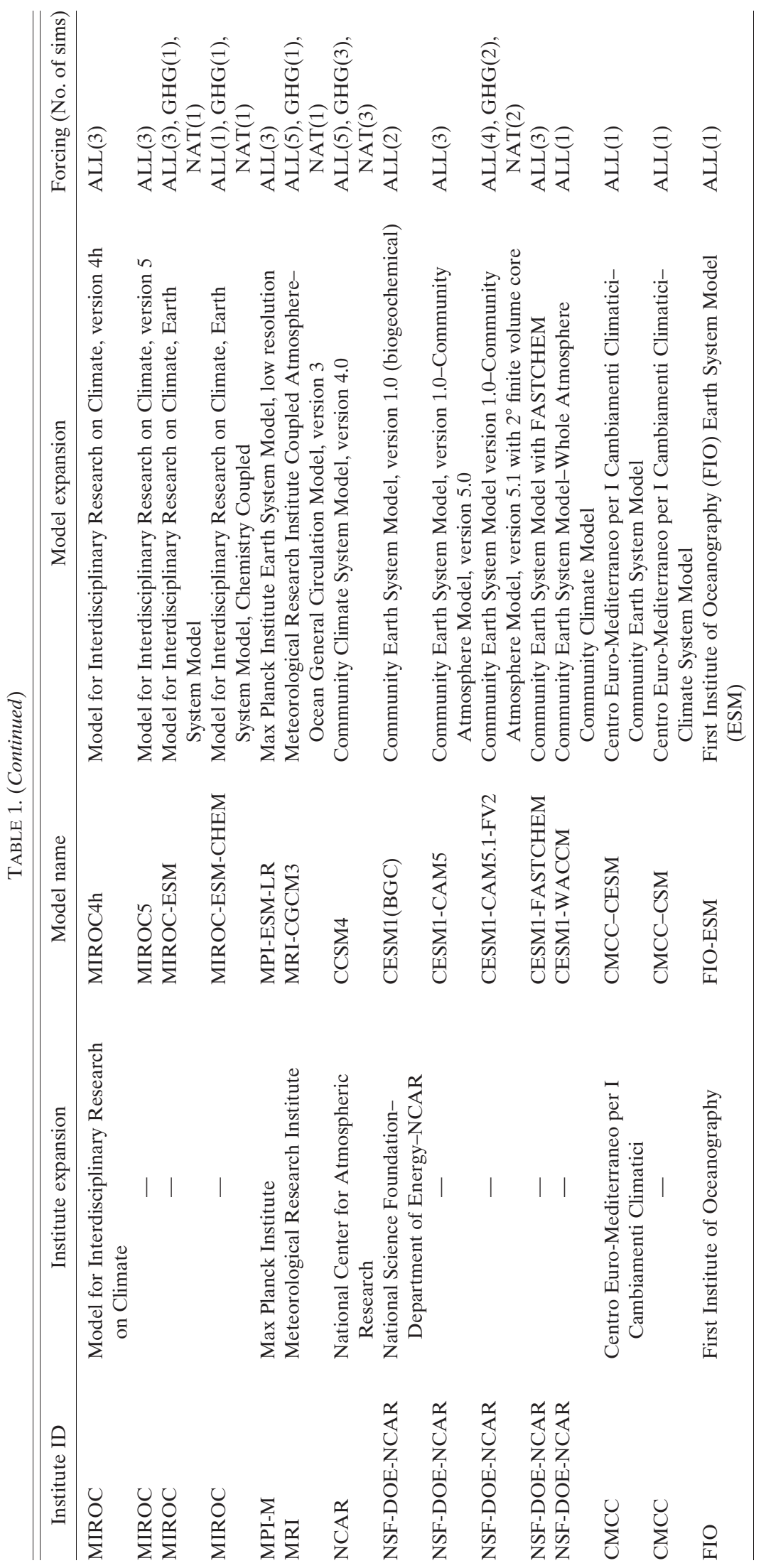



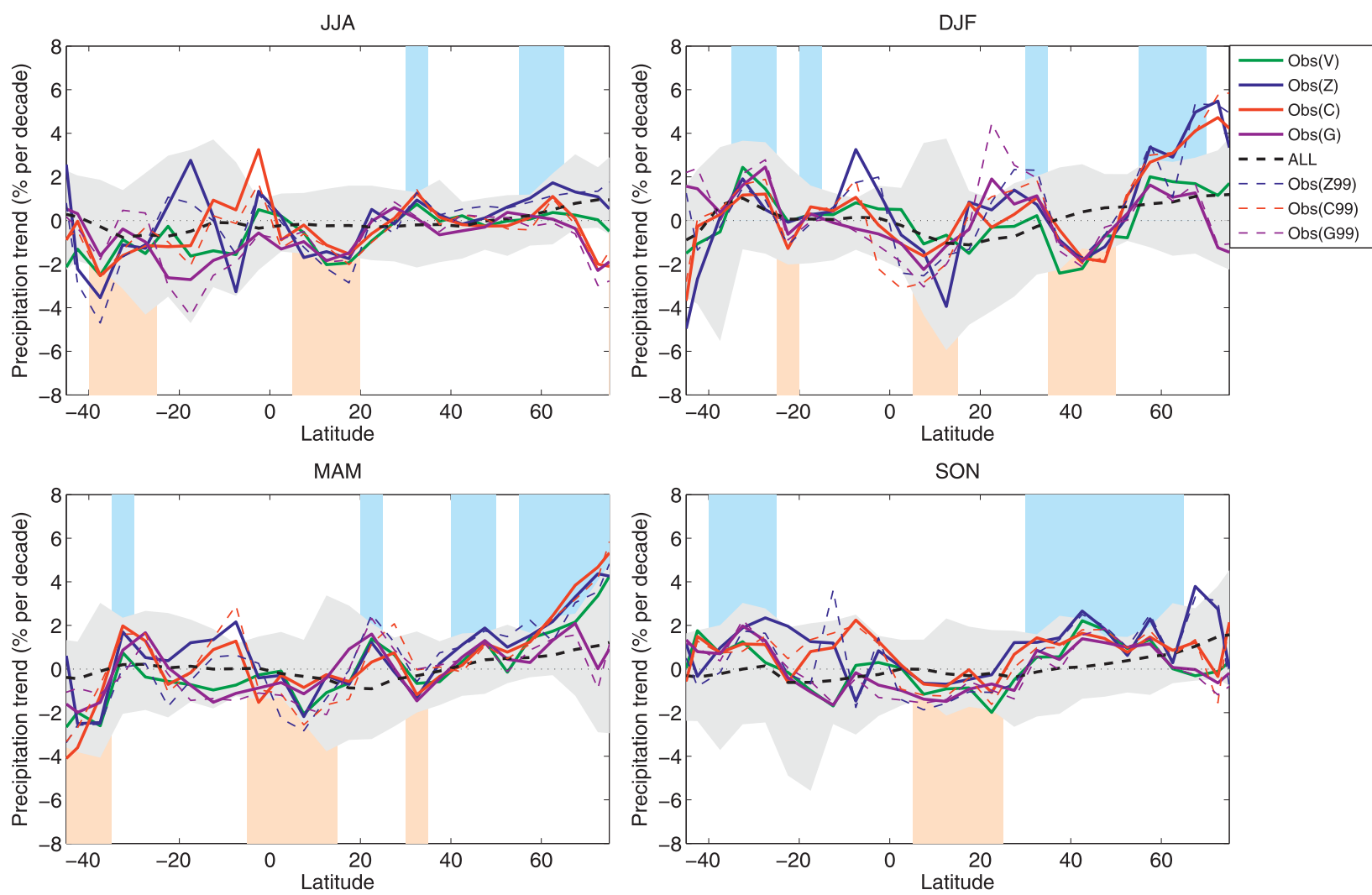

FIG. 2. Observed and multimodel-simulated seasonal zonal-mean land precipitation changes (\% decade ${ }^{-1}$ ) for the four observational datasets: CRU for 1951-2005 [Obs(C)] and 1951-99 [Obs(C99)], Zhang for 1951-2005 [Obs(Z)] and 1951-99 [Obs(Z99)], VASClimO [(Obs(V)] for 1952-2000, and GPCC for 1951-2005 [Obs(G)] and 1951-99 [Obs(G99)]. Multimodel means of the ALL-forced simulations are shown in black, the gray area is model $90 \%$ confidence interval, and blue (orange) areas show where observed changes are all positive (negative).

\section{Results}

\section{a. Role of external forcing}

Figure 2 shows the changes in precipitation in the four observed datasets, using each dataset's full coverage except for the removal of grid boxes with coverage in fewer than $95 \%$ of the years. Blue shading shows where zonal changes for all observed changes are positive $(13 \%, 29 \%, 33 \%$, and $42 \%$ of zonal bands for JJA, DJF, MAM, and SON, respectively) and the orange shading shows where they are all negative $(25 \%, 25 \%, 29 \%$, and $17 \%$ of zonal bands for JJA, DJF, MAM, and SON, respectively), yielding the most consistent changes in MAM (62\%) and the least in JJA (38\%). Despite some differences in the spatial and temporal coverage of the four observational datasets, the observational zonalchange patterns are similar with correlation coefficients between 0.4 and 0.9 for most datasets and seasons (i.e., correlation is significant with $p$ value $<0.05)$, though they are not statistically significant for the Zhang and CRU and Zhang and GPCC datasets in some seasons.
All observational datasets show increased precipitation in the Northern Hemisphere mid- to high latitudes in all seasons, drying of the Northern Hemisphere subtropics in DJF and tropics in all seasons, and drying in the Southern Hemisphere subtropics in DJF, JJA, and MAM. Where the different observational datasets disagree on the sign of the change is typically in latitude bands where the change is small. The largest disagreement between observations occurs in the Southern Hemisphere tropics, where data are spatially limited and hence changes are sensitive to even minor variations in the spatial coverage of the datasets. The magnitude of the observed changes tends to be larger in MAM and DJF, particularly in the Northern Hemisphere. To determine the influence of recent years on change patterns, changes were calculated for 1951-99 for the CRU, Zhang, and GPCC datasets and were found to be very similar with changes for 1951-2005, with correlation coefficients between 0.83 and 0.97 (see Fig. 2).

Figure 3 shows the ALL-forced multimodel mean where the models have been masked to each observational dataset for space and time. Blue shading shows where 

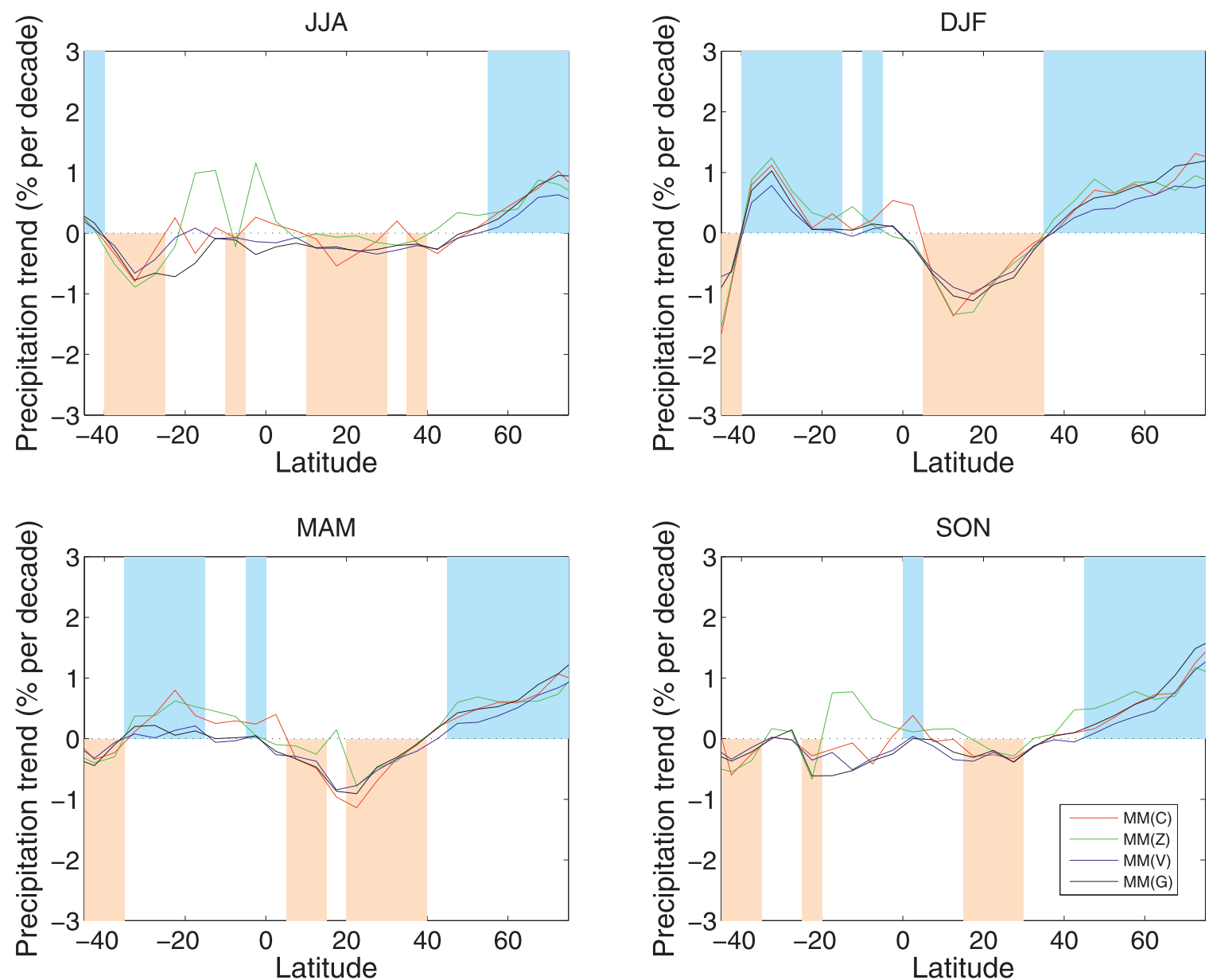

FIG. 3. Multimodel-mean zonal-mean land precipitation changes for ALL forcing ( $\%$ decade $\left.{ }^{-1}\right)$, where model data have been masked to match the different spatial and temporal coverage of the four observational datasets: CRU (1951-2005), Zhang (1951-2005), VASClimO (1952-2000), and GPCC (1951-2005) for DJF, MAM, JJA, and SON. Blue areas show where all changes are positive irrespective of masking and orange areas show where all changes are negative.

changes for all multimodel means are positive irrespective of coverage $(21 \%, 58 \%, 46 \%$, and $29 \%$ of zonal bands for JJA, DJF, MAM, and SON, respectively) and the orange shading shows where they are all negative (38\%, $29 \%, 33 \%$, and $25 \%$ of zonal bands for JJA, DJF, MAM, and SON, respectively), yielding the most consistency in MAM (87\%) and the least in SON (54\%). The zonal bands where the sign of the changes for the four observational datasets is different and not close to zero largely coincide with areas where the different masking of the same multimodel fingerprint notably modifies the outcome, suggesting that the sign differences, as well as some variations in the magnitude of the changes between observational datasets (e.g., the negative tropical change in DJF), appear to be at least partly related to data coverage. Also changes in the Southern Hemisphere tropics tend not to be the same sign for all datasets, reflecting the poorer data availability in this region.
Figure 4 shows the ALL-forced simulated changes for the individual models where the models have been masked to match the spatial and temporal coverage of the GPCC dataset, which has the largest spatial and temporal coverage. The observational changes tend to be largely within the range of simulated changes from the individual model simulations. Blue shading shows where changes for $75 \%$ of the models are positive and the orange shading shows where $75 \%$ of the models are negative. The models tend to show larger consistent changes in DJF than in other seasons and more so in the Northern Hemisphere. The most consistent response across models is an increase in precipitation in the Northern Hemisphere mid- to high latitudes in all seasons, which is also seen in the observations, particularly in DJF and MAM. The models also tend to produce a consistent pattern of decreasing precipitation at around $30^{\circ} \mathrm{N}$ in DJF and around $20^{\circ} \mathrm{N}$ in MAM, while the 

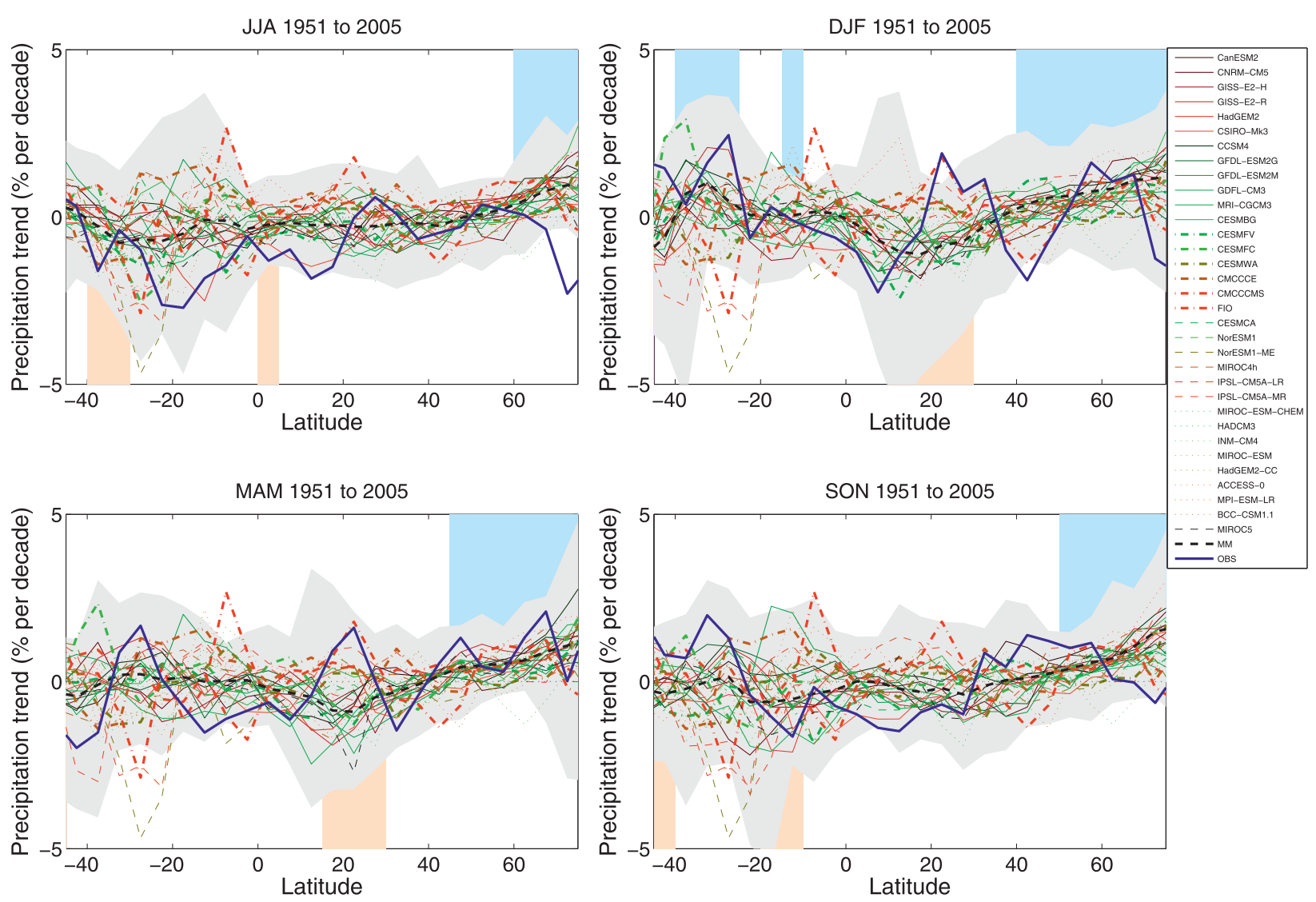

FIG. 4. Individual-model-mean and multimodel-mean (black) zonal-mean land precipitation changes for ALL forcing (\% decade ${ }^{-1}$ ), where model data have been masked to match the spatial and temporal coverage of the GPCC dataset (1951-2005) for DJF, MAM, JJA, and SON (in blue). Gray areas reflect the individual simulations' $90 \%$ confidence interval, blue areas show where $75 \%$ of models give positive changes, and orange areas show where $75 \%$ of models give negative changes.

observations consistently show moistening in these latitude bands across all datasets.

Figure 1 shows the spatial precipitation change pattern for all four observational datasets for MAM, highlighting the different spatial coverage. Figure 5 shows the spatial change patterns for the GPCC dataset for all four seasons, where the hatched areas show where all available observational datasets agree on the sign of the change. The sign of the observed changes agrees over large areas, though not all four datasets have data in these regions, but disagree in all the seasons in parts of South America and eastern and northern Asia. Figure 6 shows where all observations agree with the sign of the multimodel-mean change. The multimodel-mean change tends to be the same sign as the observations in all seasons over Europe, northern parts of North America, parts of southern Africa and South America, and Western Australia but is not the same in large parts of Asia, South America, Africa, and the United States where the observed changes are consistent across all four datasets. In most cases, for example the western
United States, parts of central Africa, and eastern Brazil in DJF, the model changes are not consistently positivenegative (i.e., $<75 \%$ of models give changes of the same sign) in these regions indicating that climate variability may be responsible for the discrepancies between the multimodel mean and the observations.

\section{b. Role of individual external forcings}

Figure 7 shows the ALL, GHG, ANT, NAT, and AA multimodel simulated changes masked to the GPCC dataset. The magnitude of the changes for the NATforced multimodel mean tends to be smaller than that for the other forcings, and the ensemble encompasses zero for all latitude bands. The blue areas show where ALL-, GHG-, and ANT-forced changes are all positive, orange shows where they are all negative, and the $x$ symbols show where over $75 \%$ of the simulations give a change of the same sign. The changes are the same sign for the different forcings in the mid- and high latitudes, particularly in the Northern Hemisphere where all show increased precipitation. It is also at these latitudes that 

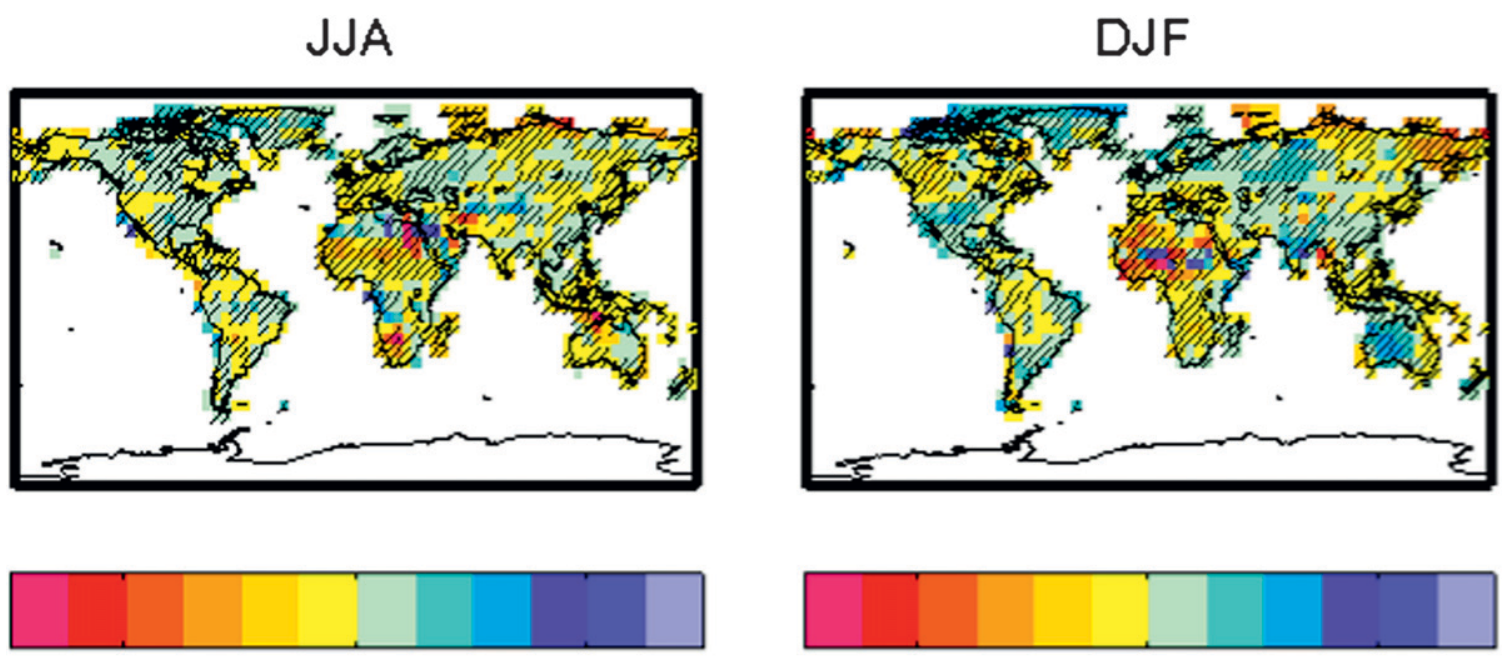

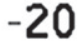

0 MAM

20
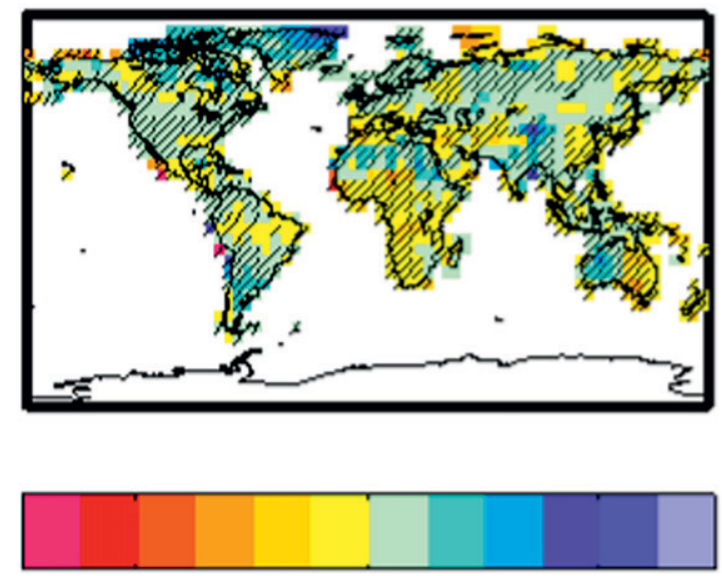

$-20$

20
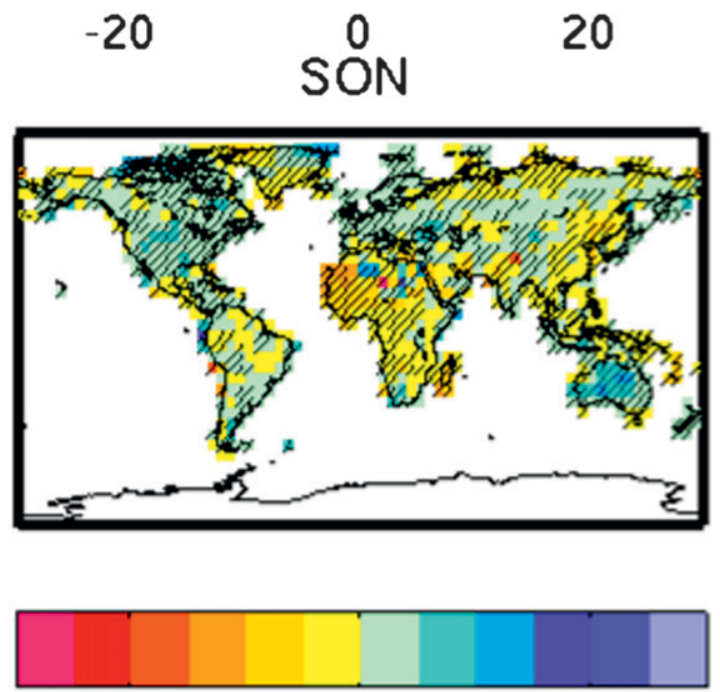

$-20$

FIG. 5. Percentage change in precipitation per decade for the GPCC dataset for 1951-2005 for DJF, MAM, JJA, and SON. Hatched grid boxes show where the sign of the change is consistent across all observation datasets with data available for that grid box.

the changes of the individual simulations tend to be most consistent ( $>75 \%$ give changes of the same sign). These three different forcings tend to produce changes with different signs in the tropics and subtropics. Across much of this region the precipitation change from the ALL-forced multimodel mean is negative while the change from the GHG- and ANT-forced simulations is more structured. The changes for the NAT-forced simulations tend to be less consistent overall than for the other forcings, with only a few zonal bands with over $75 \%$ of simulations giving a change of the same sign and none at all in MAM or SON. Natural forcing over that period is limited to very small changes in solar forcing and a few volcanic eruptions whose effect, particularly on land precipitation, should not lead to long-term trends.
Figures S2-S6 in the supplemental material show the zonal-mean changes from observations plotted against the multimodel-mean changes for each latitude for ALL, GHG, NAT, ANT, and AA forcings, respectively. The observations show the least agreement with only NAT or only AA forcings and show the most agreement for the ALL-forced multimodel mean.

Figure 8 shows the spatial precipitation patterns of change of the multimodel means for MAM for each of the different forcings, ALL, GHG, NAT, ANT, and AA. The patterns of change are largely consistent for the multimodel means of ALL-, GHG-, and ANT-forced simulations. This is consistent with GHG being the largest forcing over the past $50 \mathrm{yr}$. In contrast, the NAT- and AA-forced simulations have distinctly different patterns 

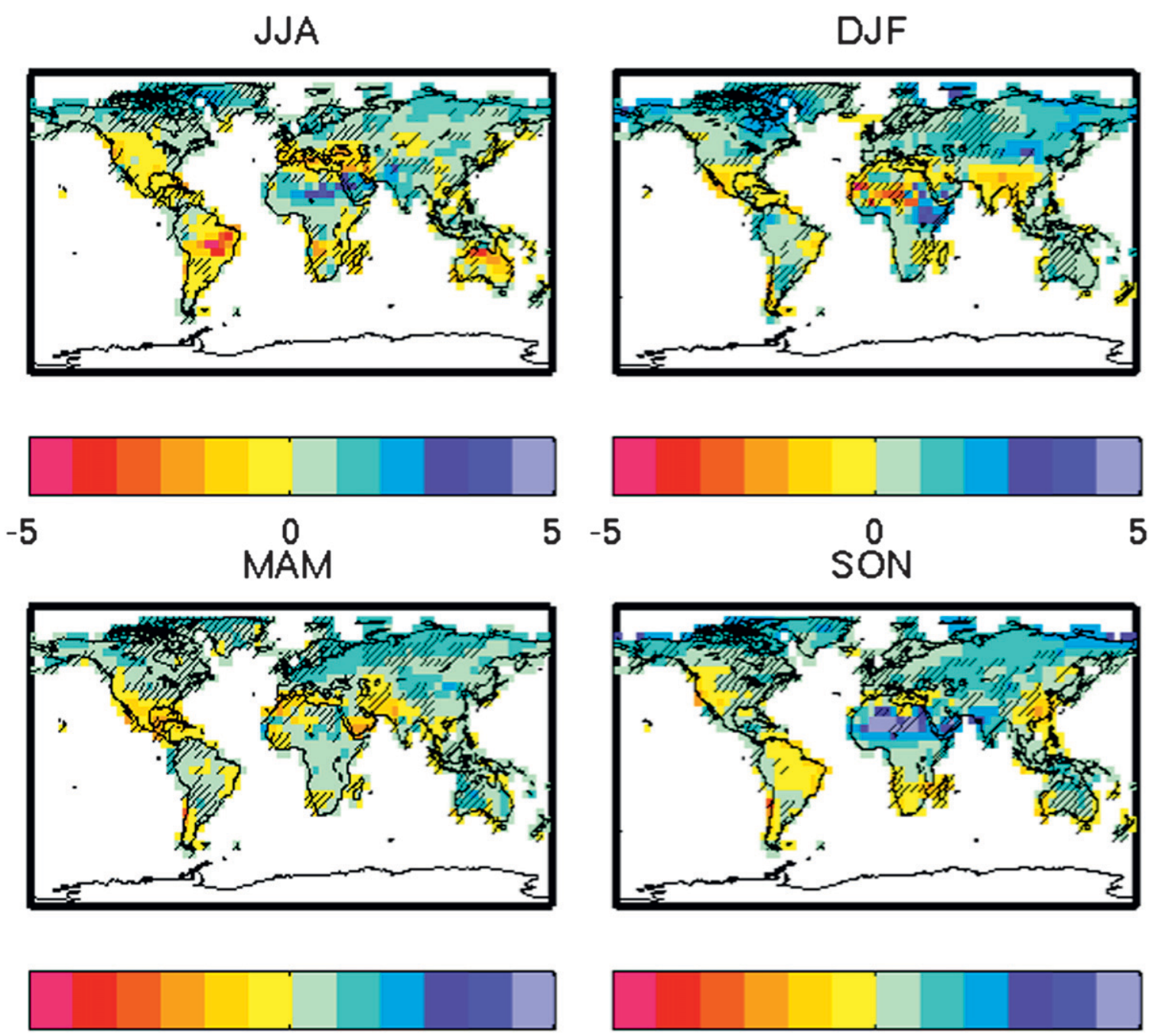

$-5$

0

$5 \quad-5$

5

FIG. 6. Percentage change in precipitation per decade for the ALL-forced multimodel mean for 1951-2005 for DJF, MAM, JJA, and SON. Hatched grid boxes show where the sign of the change is consistent across all four observation datasets and the multimodel mean. Note the smaller scale of change patterns as multimodel-mean changes show a much reduced influence of internal climate variability.

of moistening and drying; for example, the NAT and AA changes show moistening over southern Europe, while the ALL, GHG, and ANT changes all show drying. Conversely, in large parts of Asia the NAT and AA forcing, according to the models, should have caused drying while the ALL, GHG, and ANT changes show an increase in precipitation. Figure 8 also shows the multimodel mean of the ALL-forced simulations from CMIP3. The sign of the changes is largely the same for large parts of the globe for CMIP3 and CMIP5 multimodel means; however, there are some differences over parts of Africa, South America, and Asia with the CMIP5 changes tending to show an increase in precipitation compared to a decrease from CMIP3. However, these differences tend to be small and in regions where there is no consistency between the sign of the changes in the CMIP5 models (i.e., sign of change is the same in $<75 \%$ of models); therefore, the differences are likely to be explained, at least in part, by the different composition of models in the CMIP3 and CMIP5 ensembles.

\section{c. Results of detection and attribution}

We will now discuss the results of a detection and attribution method aiming to attribute causes to the observed changes. Figure 9 shows the scaling factors and $90 \%$ confidence intervals for all four datasets for the ALL-forced simulations, GHG-forced simulations, NATforced simulations, and ANT- and AA-forced simulations, and Table 2 shows the scaling factors and $90 \%$ confidence interval for the ALL-forced simulations. For 

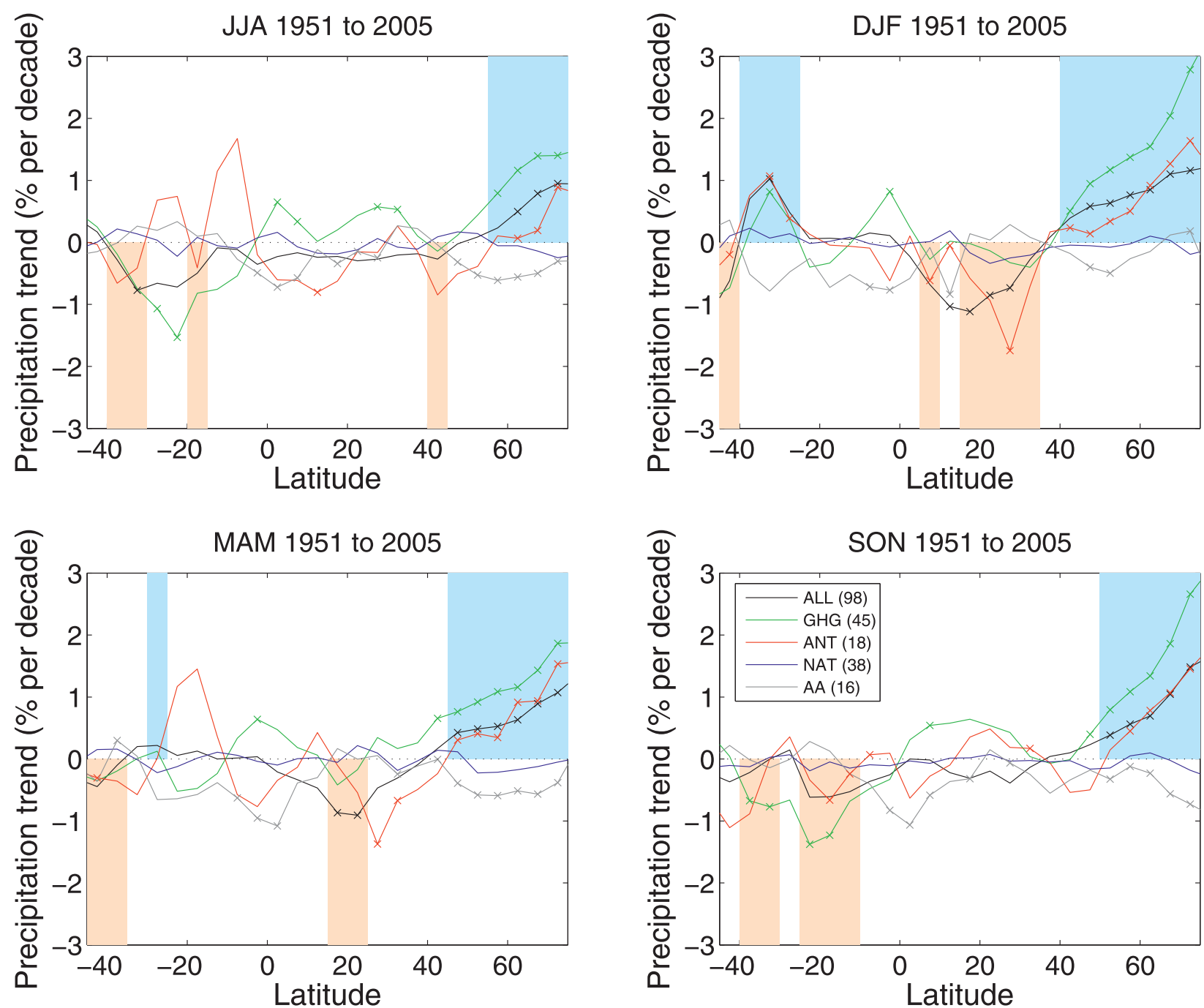

FIG. 7. Multimodel-mean zonal-mean land precipitation changes for ALL, GHG, ANT, NAT, and AA forcings ( $\%$ decade $\left.{ }^{-1}\right)$, where model data have been masked to matched the spatial and temporal coverage of the GPCC dataset (1951-2005) for DJF, MAM, JJA, and SON. The blue areas show where the changes of the multimodel mean of the ALL, GHG, and ANT forcings are all positive and the orange areas show where they are all negative. The $\times$ symbols show where over $75 \%$ of the simulations give changes of the same sign.

the ALL and ANT fingerprints, a detectable result at the $5 \%$ significant level for double the variance is found for three datasets in MAM, DJF, and annual (ANN). (By doubling the variance of the model-based estimates of internal variability, we allow for the possible underestimation of precipitation variability by the models and obtain a more conservative-i.e., wider-confidence interval.) Only GPCC (shown in gray), which has not been homogenized for trend analysis, does not show a detectable signal of external forcing; however, changes are detectable for the VASClimO dataset, a homogenized subset of the GPCC dataset. In that case, the $90 \%$ confidence interval is very close to 0 for DJF for double the variance, so the forcing signal may only just be emerging from the climate noise for VASClimO in this season. Only the Zhang dataset shows detectable changes for all four seasons, even if the variance of noise is doubled. GHG forcing is detected for the same datasets and seasons as ALL forcings, except for VASClimO in DJF and ANN and CRU in ANN. Neither NAT nor AA forcing are detected consistently across any dataset or season; however, the $90 \%$ confidence intervals are negative for three datasets in MAM (i.e., the pattern of change for AA forcing is opposite that observed). This is consistent with GHG forcing being primarily responsible for the forced changes in the ALL and ANT fingerprints detected in the observations.

For ALL forcing, the scaling factor is not consistently greater than 1 for any season or for annual changes, and the best-guess scaling factors are largely in the range 


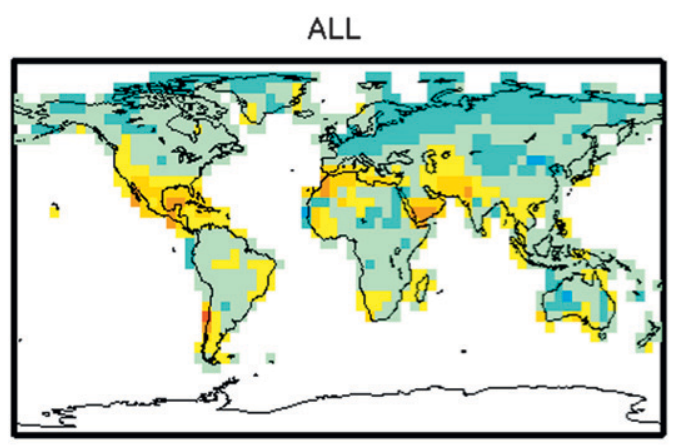

NAT

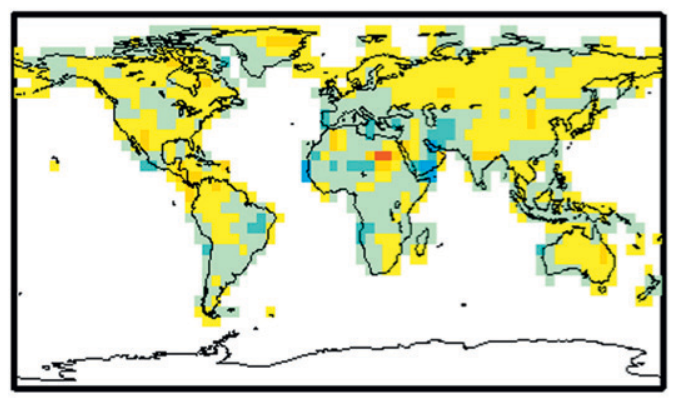

AA

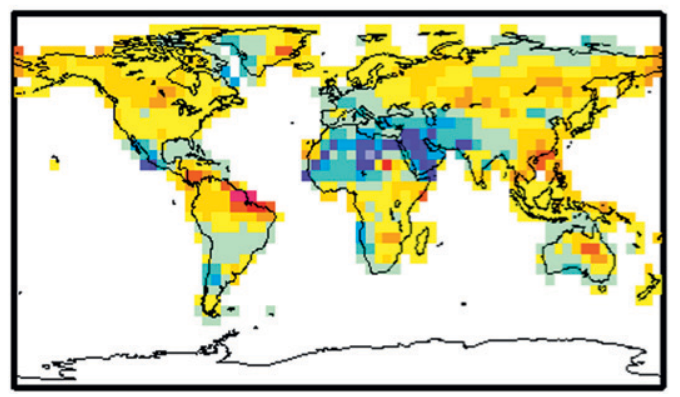

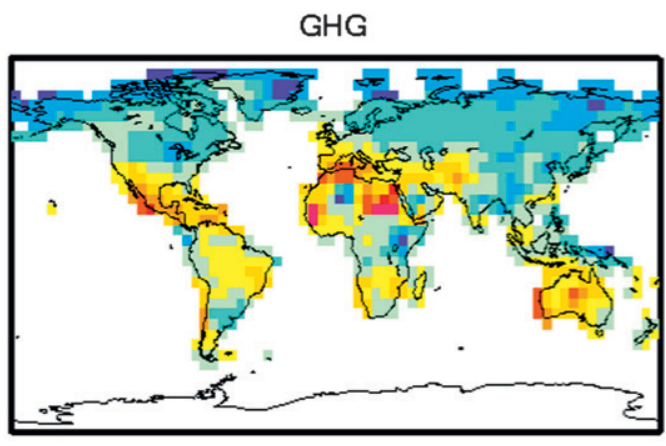

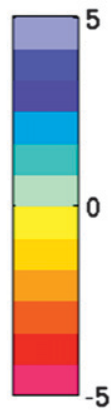

ANT

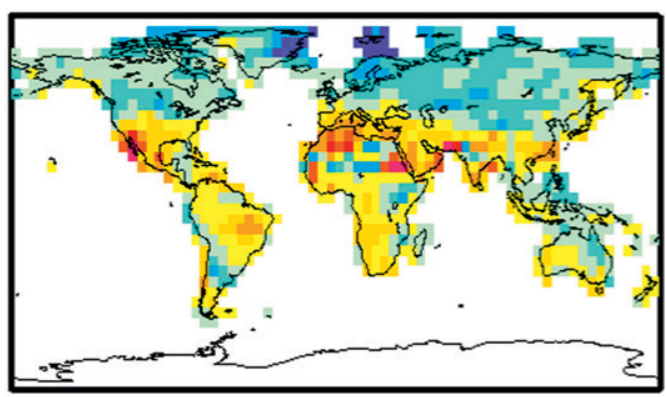

ALL CMIP3

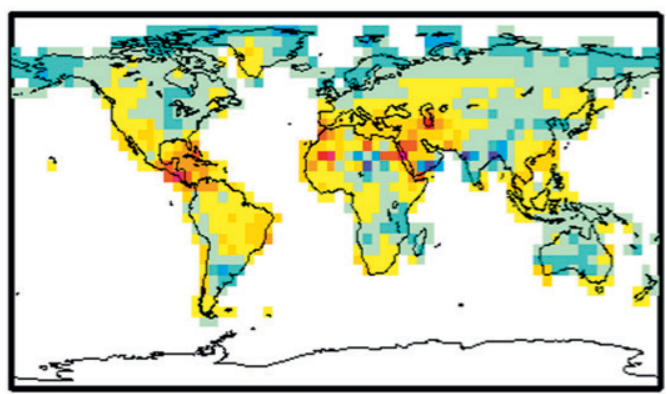

FIG. 8. Percentage change in precipitation per decade for 1951-2005 for MAM for the multimodel mean of ALL (98), GHG (45), ANT (18), NAT (38), and AA-forced (16) simulations, and the multimodel mean of the ALL-forced (54) simulations from CMIP3.

$0-2$. Thus, the magnitude of the precipitation changes is largely captured by the models when expressed as percentage change relative to the internal climatology of the model or observational dataset. The results for the ANT-forced fingerprint are mostly similar to those of the ALL forcing, as are those for GHG-only forcing.

Scaling factors for the two-signal analysis are shown in Fig. 10, which determines if the response to individual forcing can be distinguished from each other. For the two-signal analysis, no forcing is detected consistently across all datasets at the $5 \%$ level of significance for double the variance. However, GHG forcing is detectable for the Zhang dataset at the $5 \%$ level of significance when AA forcing is included separately into the analysis and for ANT forcing when including NAT forcing separately.

The residuals after tls fitting are compared to the variance in the simulations using an $F$ test to ensure that the residuals are not significantly different from those expected from control simulations (Allen and Stott 2003). Where the $F$ test fails either the regression does not contain all relevant and realistic response fingerprints to external forcings or the model variability is erroneous. The residual consistency test is passed for all forcings and seasons when the model variance is doubled.

\section{Discussion and conclusions}

In Noake et al. (2012), the datasets and models were masked to have the same spatial and temporal coverage as each other. Here, the observational datasets have not been masked except to remove grid boxes with poor temporal coverage, and the simulations have been masked to each dataset individually to derive fingerprints. 

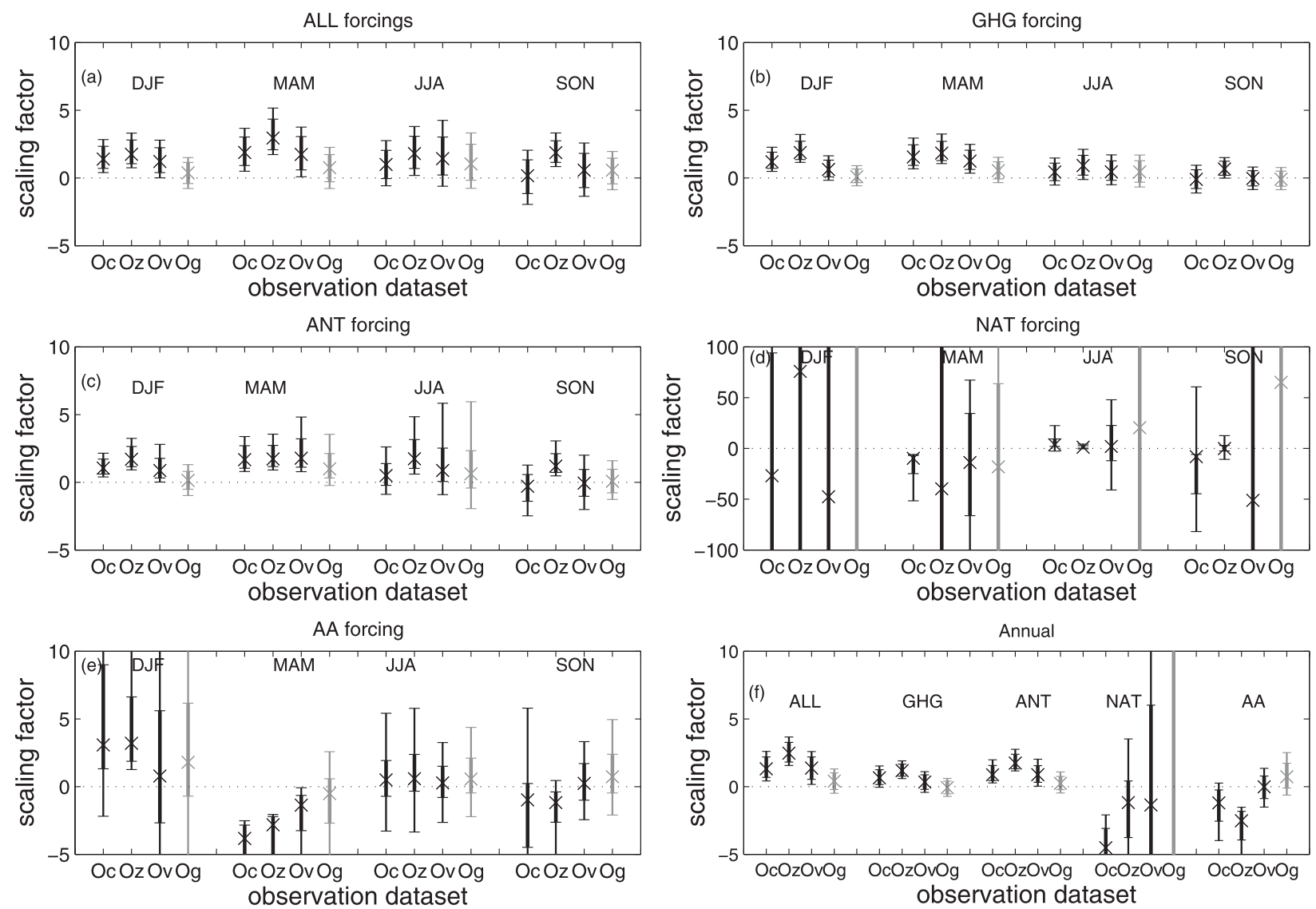

FIG. 9. Detection results for individual fingerprints. Scaling factors [see Eq. (1)] are given for (a)-(e) seasonal and (f) annual changes for CRU (Oc) for 1951-2005, Zhang (Oz) for 1951-2005, VASClimO (Ov) for 1952-2000, and GPCC (Og) for 1951-2005 observations. (a) ALL-, (b) GHG-, (c) ANT-, (d) NAT-, and (e) AA-forced simulation-based fingerprints. (f) Scaling factors for annual changes for all five fingerprints. The $\times$ symbols show the "best-guess" scaling factor for the multimodel mean, thick lines are the $90 \%$ confidence interval for the raw variance added as noise, and thin lines are the $90 \%$ confidence interval for double the variance.

Nevertheless, the resulting change patterns are similar for all observational datasets, despite differences in data coverage. The Southern Hemisphere tropics are an exception, as spatial coverage is particularly poor because of the limited availability of land station data (see, e.g., Becker et al. 2013). In these regions, ocean data are required to improve the calculation of zonal-mean precipitation changes, but the relatively short satellite data coverage means that the signal-to-noise ratio of these data may be too low to allow for the detection of forcing using this approach. However, there is evidence from satellite and model data that land and ocean precipitation in the tropics and globally are negatively correlated (Gu et al. 2007; Liu et al. 2012), while analysis of salinity data suggests that an enhanced $P-E$ signal may also be emerging there (Durack et al. 2012). We find that differences in spatial coverage play an important role in differences in zonal-mean changes between datasets.

The zonal patterns of change are similar for the ALL, ANT, and GHG forcing at latitude bands where over

TABLE 2. Scaling factors for tls detection and attribution for ALL forcing. Table shows best-fit scaling factor and $90 \%$ confidence interval in square brackets for double the variance. Values in bold show where forcing is detected.

\begin{tabular}{lccccc}
\hline \hline Obs dataset & \multicolumn{1}{c}{ DJF } & \multicolumn{1}{c}{ MAM } & JJA & SON & Annual \\
\hline CRU & $\mathbf{1 . 4 0}[\mathbf{0 . 3 9}-\mathbf{2 . 8 3}]$ & $\mathbf{1 . 8 8}[\mathbf{0 . 5 0}-\mathbf{3 . 6 6}]$ & $1.00[-0.58-2.76]$ & $0.16[-1.95-2.04]$ & $\mathbf{1 . 3 2}[\mathbf{0 . 4 1 - 2 . 6 0}$ \\
Zhang & $\mathbf{1 . 7 5}[\mathbf{0 . 7 5}-\mathbf{3 . 3 1}]$ & $\mathbf{2 . 9 4}[\mathbf{1 . 7 2 - 5 . 1 6}]$ & $\mathbf{1 . 8 0}[\mathbf{0 . 1 8}-\mathbf{3 . 7 9}]$ & $\mathbf{1 . 8 6}[\mathbf{0 . 8 4 - 3 . 3 2}]$ & $\mathbf{2 . 4 6}[\mathbf{1 . 5 6}-\mathbf{3 . 6 6}]$ \\
VASClimO & $\mathbf{1 . 2 2}[\mathbf{0 . 0 0 7 - 2 . 7 9 ]}$ & $\mathbf{1 . 7 4}[\mathbf{0 . 0 8}-\mathbf{3 . 7 6}]$ & $1.42[-0.62-4.26]$ & $0.57[-1.35-2.57]$ & $\mathbf{1 . 3 7}[\mathbf{0 . 1 6}-\mathbf{2 . 5 9}]$ \\
GPCC & $0.38[-0.78-1.51]$ & $0.76[-0.78-2.26]$ & $1.04[-0.77-3.323]$ & $0.57[-0.87-1.96]$ & $0.40[-0.49-1.30]$ \\
\hline
\end{tabular}



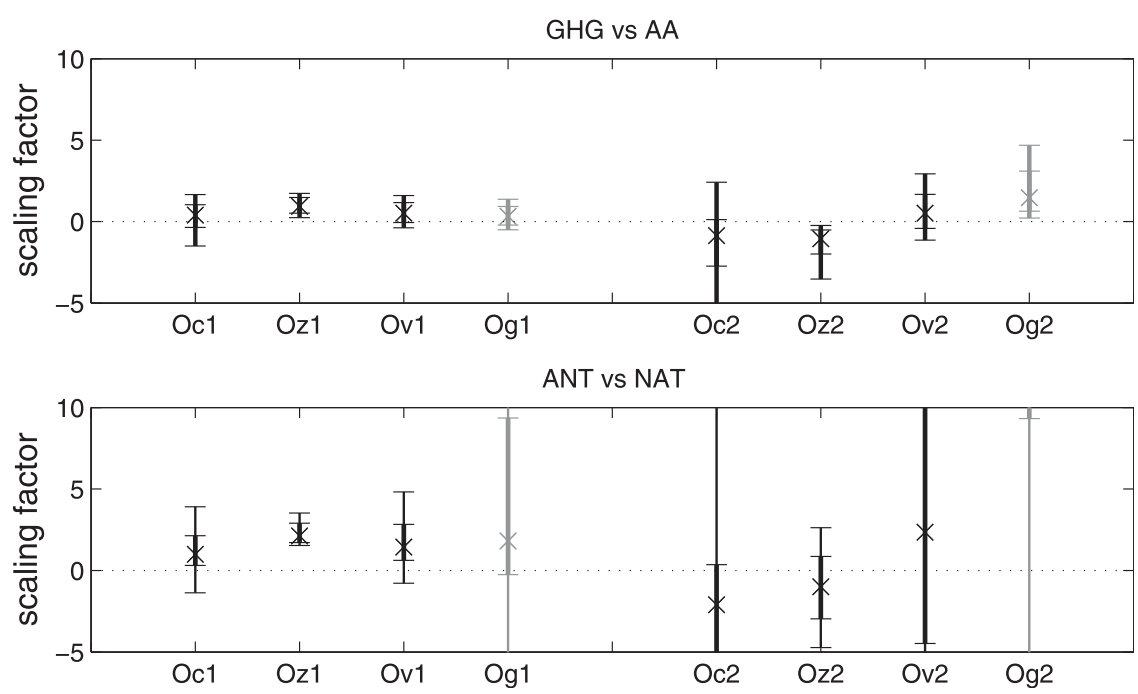

FIG. 10. Scaling factors two-signal fingerprint detection for (top) GHG(Ox1) and AA(Ox2) and (bottom) ANT $(\mathrm{O} x 1)$ and $\mathrm{NAT}(\mathrm{O} x 2)$ forced simulation-based fingerprints for CRU $(\mathrm{Oc})$ for 1951-2005, Zhang (Oz) for 1951-2005, VASClimO (Ov) for 1952-2000, and GPCC (Og) for 1951-2005 observations for annual changes. The $\times$ symbols show the best-guess scaling factor for the multimodel mean, thick lines are the $90 \%$ confidence interval for the raw variance added as noise, and thin lines are the $90 \%$ confidence interval for double the variance.

$75 \%$ of the simulations give changes of the same sign. In particular in the Northern Hemisphere mid- and high latitudes, where land coverage and hence spatial coverage is greatest, the changes for ALL, GHG, and ANT are all positive while the changes for AA are negative. The smaller positive changes for ALL and ANT forcing compared to the GHG forcing are consistent with the negative influence of AA forcing on the changes in precipitation at these latitude bands. Aerosols affect the climate by direct absorption of radiation and indirectly by changing cloud properties. Emissions of aerosols are greater in the Northern Hemisphere. Hence, because of their relatively short lifetimes in the atmosphere, aerosols have a stronger cooling effect on in the Northern Hemisphere. The spatial pattern of AA-induced changes shows a decrease in precipitation over much of the United States, Europe, and Asia. Anthropogenic aerosol emissions have been shown to suppress monsoon circulation and cause a reduction in precipitation over South and East Asia (Randles and Ramaswamy 2008; Bollasina et al. 2011; Guo et al. 2012) and to change extratropical circulation with associated changes in patterns of precipitation (Ming et al. 2011).

Understanding the physical mechanisms responsible for the patterns of change in the zonal-mean precipitation is difficult because of a number of complicating factors such as poor spatial coverage in some zonal bands, the influence of natural variability (which will have a greater impact where spatial coverage is limited), and the fact that we are average over different circulation regimes. As a first step we consider the $P-E$ patterns of change from Held and Soden (2006). These are dominated by changes in precipitation so we might expect that the zonal patterns of precipitation change would match those of $P-E$ at least qualitatively in terms of the sign of the change; however, enhanced $P-E$ will not apply as well for the land-only zonal means used here, so some disagreement is expected. The ALL-forced multimodel-mean changes in precipitation are the same sign as the $P-E$ patterns in the Northern Hemisphere subtropics and mid- and high latitudes but not the tropics or Southern Hemisphere. The GHG- and ANTforced multimodel mean are similar, except that small $\left(<1 \%\right.$ decade $\left.^{-1}\right)$ positive changes occur in Northern Hemisphere subtropical precipitation in JJA and SON. The increase in observed precipitation in the Northern Hemisphere high latitudes is consistent with increasing moisture transport into these regions (Seager et al. 2010; Bengtsson et al. 2011), while the decrease in precipitation in the Northern Hemisphere subtropics is consistent with the expected expansion of the subtropical dry regions as a result of the expansion of the Hadley cell (Lu et al. 2007; Seager et al. 2010; Scheff and Frierson 2012) and poleward shift of the midlatitude storm tracks, which transport moisture poleward from the subtropics (Yin 2005; Lorenz and DeWeaver 2007; Seidel et al. 2008).

The zonal-mean patterns do not show the enhancement of precipitation predicted in the zonal tropics by Held and Soden (2006). In the tropics, precipitation is 
enhanced over convergence regions and decreases in subsidence regions (due to increased moisture transport), though dynamical feedbacks can produce changes of the opposite sign within these regions (Chou and Neelin 2004; Neelin et al. 2006; Chou et al. 2009). Seager et al. (2010) show from modeling studies of the twentyfirst century that while changes in $P-E$ are positive in the tropics overall, changes over land are mostly negative in April-September and a mixture of positive and negative in October-March. These patterns are largely due to the increase of the advection of water vapor by the mean flow, but changes to the mean circulation dynamics are also important. Neelin et al. (2006) also show drying over tropical land in JJA in the twenty-first century and Liu et al. (2012) show that precipitation decreases with temperature over tropical land in CMIP5 models and satellite data. In the Southern Hemisphere, the lack of ocean data is particularly problematic when calculating the zonal-mean changes due to poor data coverage compared to the Northern Hemisphere, particularly in the tropics. In the subtropics, the expected drying is only seen in JJA, with increasing precipitation in DJF and SON mostly due to changes over Australia, which matches the increase in $P-E$ seen in OctoberMarch in Seager et al. (2010).

The spatial change patterns are similar for the different observational datasets in terms of the sign of change. In many regions, and in many areas where the sign of change of the multimodel mean is not the same as observations, the individual model simulations do not produce consistent moistening or drying either, emphasizing large variability in precipitation. However, in some regions observed changes are the same sign across all datasets but are different for modeled changes that are consistent across the simulations; for example, northeast Asia in DJF. Internal climate variability may explain some of the discrepancies. Dai (2013) showed how internal variability could produce apparent trends in regional precipitation on multidecadal time scales in the southwest United States while Kelley et al. (2012) show that the winter drying seen in the Mediterranean observations may be due to multidecadal variability with the radiatively forced signal only beginning to emerge from the natural variability. Regional precipitation changes will also be affected by even subtle shifts in atmospheric circulation. For example, Noake et al. (2012) investigated whether the North Atlantic Oscillation (NAO) could explain aspects of the observed changes but reveal only a small effect on the observed zonalmean changes. In contrast, the NAO affects changes in Europe at decadal time scales (Sutton and Dong 2012).

Noake et al. (2012) showed that using percentage change rather than absolute changes the model simulations tended to capture the magnitude of change for ALL-forced simulations with only MAM being consistently underestimated by the models and that the external forcing was detectable for DJF, MAM, and SON using the CMIP3 models. Here, we find a similar result using the CMIP5 models and extending the time period to 2005 for the CRU, Zhang, and the GPCC (not used in Noake et al. 2012) datasets, with the only major change being that the ALL forcing is no longer detected for the CRU and VASClimO datasets for SON and that the magnitude of change is no longer consistently underestimated in MAM for all observational datasets. In Noake et al. (2012), all datasets were masked to limit coverage to grid boxes where station data were available. However, here we include grid boxes where data have been interpolated, introducing more uncertainty but allowing for greater spatial and more consistent temporal coverage. Applying tls detection to a number of datasets and for different seasons increases the likelihood that we will obtain false positive detection results simply by chance. Only when forcing is detected for multiple datasets in the same seasons or across seasons do we have confidence that detection of forcing is robust. Robust detection results were found for MAM, DJF, and annual, where ALL forcing is detected for all datasets except the GPCC dataset, which has not been homogenized for use in change analysis. It is also worth noting that for the Zhang dataset, which is restricted to only grid boxes that contain long-term station data, we detect ALL forcing across all seasons and for annual changes as was the case in Zhang et al. (2007) for annual data.

The present study further extends the work of Noake et al. (2012) by obtaining results for individual forcings. We find that fingerprints that include the effects of greenhouse gas increases (ALL, ANT, and GHG) are similarly detectable, with changes in DJF and MAM detectable in at least two datasets even when the modelbased estimate of internal-variability variance is doubled. Neither NAT forcing nor AA forcing are detectable. AA shows negative detectable signals in MAM in three datasets, which are possibly due to aerosol forcing partly counteracting greenhouse gas forcing, possibly by precipitation response to aerosol-induced cooling (see Allen and Ingram 2002).

The two-signal detection fails to produce a detectable forcing consistently across all datasets if estimating the GHG and AA fingerprints separately or the ANT and NAT forcings separately. However, anthropogenic forcing is detected separately from natural forcing, and greenhouse gas forcing is detected separately from anthropogenic aerosol forcing for the Zhang dataset. For anthropogenic forcing, the magnitude of the scaling factor is inconsistent with " 1 " indicating that the model 
response needs to be enhanced to reproduce the observations, as was found in Zhang et al. (2007) for annual data. Overall results of the detection and attribution for global land precipitation using fingerprints from the CMIP5 models are consistent with older work using the CMIP3 models. The results confirm that external forcing had a detectable influence on seasonal land precipitation in MAM, DJF, and annual land precipitation and suggests, with uncertainties, that the externally forced changes are largely the result of human influence, particularly greenhouse gas forcing of the climate.

Acknowledgments. The authors acknowledge the use of precipitation data of the VASClimO project and Global Precipitation Climatology Centre and the contributions of David Lister and Ian Harris at the Climatic Research Unit for their assistance with producing the new version of the CRU TS precipitation dataset used here. We acknowledge the World Climate Research Programme's Working Group on Coupled Modelling, which is responsible for CMIP, and we thank the climate modeling groups (listed in Table 1) for producing and making available their model output. For CMIP, the U.S. Department of Energy's Program for Climate Model Diagnosis and Intercomparison provides coordinating support and led the development of software infrastructure in partnership with the Global Organization for Earth System Science Portals. This work was supported by the NERC Project PAGODA (Grant Number NE/I006141/1), the National Science Foundation (Grant Number ATM-0296007), NCAS, the U.S. Department of Energy's Office of Science, and NOAA's Climate Program Office. We also thank three anonymous reviewers for their insightful and helpful suggestions.

\section{REFERENCES}

Allen, M. R., and S. F. B. Tett, 1999: Checking for model consistency in optimal fingerprinting. Climate Dyn., 15, 419-434.

_ climate and the hydrologic cycle. Nature, 419, 224-232.

— fingerprinting. Part I: Theory. Climate Dyn., 21, 477-491.

Balan Sarojini, B., P. E. Stott, E. Black, and D. Polson, 2012: Fingerprints of changes in annual and seasonal precipitation from CMIP5 models over land and ocean. Geophys. Res. Lett., 39, L21706, doi:10.1029/2012GL053373.

Beck, C., J. Grieser, and B. Rudolf, 2005: A new monthly precipitation climatology for the global land areas for the period 1951 to 2000. Klimastatusbericht 2004, DWD, 181-190. [Available online at http://www.dwd.de/bvbw/generator/DWDWWW/Content/ Oeffentlichkeit/KU/KU4/KU42/en/VASClimO/pdf_28_ precipitation, templateId $=$ raw, property $=$ publicationFile. pdf/pdf_28_precipitation.pdf.]

Becker, A., and Coauthors, 2013: A description of the global landsurface precipitation data products of the Global Precipitation
Climatology Centre with sample applications including centennial (trend) analysis from 1901-present. Earth Syst. Sci. Data, 5, 71-99.

Bengtsson, L., K. I. Hodges, S. Koumoutsaris, M. Zahn, and N. Keenlyside, 2011: The changing atmospheric water cycle in polar regions in a warmer climate. Tellus, 63A, 907-920.

Bollasina, M. A., Y. Ming, and V. Ramaswamy, 2011: Anthropogenic aerosols and the weakening of the South Asian summer monsoon. Science, 334, 502-505.

Cayan, D. R., M. D. Dettinger, H. F. Diaz, and N. E. Graham, 1998: Decadal variability of precipitation over western North America. J. Climate, 11, 3148-3166.

Chou, C., and J. D. Neelin, 2004: Mechanisms of global warming impacts on regional tropical precipitation. J. Climate, 17, 2688-2701.

,-- C. Chen, and J. Tu, 2009: Evaluating the "rich-getricher" mechanism in tropical precipitation change under global warming. J. Climate, 22, 1982-2005.

Dai, A., 2013: The influence of the inter-decadal Pacific oscillation on U.S. precipitation during 1923-2010. Climate Dyn., 41 (3-4), 633-646, doi:10.1007/s00382-012-1446-5.

Durack, P. J., S. E. Wijffels, and R. J. Matear, 2012: Ocean salinities reveal strong global water cycle intensification during 1950 to 2000. Science, 336, 455-458.

Gillett, N. P., A. J. Weaver, F. W. Zwiers, and M. F. Wehner, 2004: Detection of volcanic influence on global precipitation. Geophys. Res. Lett., 31, L12217, doi:10.1029/2004GL020044.

Gu, G., R. F. Adler, G. J. Huffman, and S. Curtis, 2007: Tropical rainfall variability on interannual-to-interdecadal and longer time scales derived from the GPCP monthly product. J. Climate, 20, 4033-4046.

Guo, L., E. J. Highwood, L. C. Shaffrey, and A. G. Turner, 2012: The effect of regional changes in anthropogenic aerosols on rainfall of the East Asian summer monsoon. Atmos. Chem. Phys. Discuss., 12, 23 007-23038.

Harris, I., P. D. Jones, T. J. Osborn, and D. H. Lister, 2013: Updated high-resolution grids of monthly climatic observationsThe CRU TS3.10 dataset. Int. J. Climatol., in press.

Hegerl, G. C., T. J. Crowley, M. R. Allen, W. T. Hyde, H. N. Pollack, J. Smerdon, and E. Zorita, 2007: Detection of human influence on a new, validated 1500-year temperature reconstruction. J. Climate, 20, 650-666.

Held, I. M., and B. J. Soden, 2006: Robust responses of the hydrological cycle to global warming. J. Climate, 19, 5686-5699.

Hoerling, M., J. Hurrell, J. Eischeid, and A. Phillips, 2006: Detection and attribution of twentieth-century northern and southern African rainfall change. J. Climate, 19, 3989-4008.

_ J. Eischeid, J. Perlwitz, X. Quan, T. Zhang, and P. Pegion, 2012: On the increased frequency of Mediterranean drought. J. Climate, 25, 2146-2161.

Huffman, G. J., R. F. Adler, D. T. Bolvin, and G. Gu, 2009: Improving the global precipitation record: GPCP version 2.1. Geophys. Res. Lett., 36, L17808, doi:10.1029/2009GL040000.

Ineson, S., and A. A. Scaife, 2009: The role of the stratosphere in the European climate response to El Niño. Nat. Geosci., 2, 32-36.

Jones, P. D., and M. Hulme, 1996: Calculating regional climatic time series for temperature and precipitation: Methods and illustrations. Int. J. Climatol., 16, 361-377.

Kang, S. M., L. M. Polvani, J. C. Fyfe, and M. Sigmond, 2011: Impact of polar ozone depletion on subtropical precipitation. Science, 332, 951-954.

Kelley, C., M. Ting, R. Seager, and Y. Kushnir, 2012: Mediterranean precipitation climatology, seasonal cycle, and trend as 
simulated by CMIP5. Geophys. Res. Lett., 39, L21703, doi:10.1029/2012GL053416.

Kenyon, J., and G. C. Hegerl, 2010: Influence of modes of climate variability on global precipitation extremes. J. Climate, $\mathbf{2 3}$, 6248-6262.

Knutti, R., R. Furrer, C. Tebaldi, J. Cermak, and G. A. Meehl, 2010: Challenges in combining projections from multiple climate models. J. Climate, 23, 2739-2758.

Lambert, F. H., and M. R. Allen, 2009: Are changes in global precipitation constrained by the tropospheric energy budget? J. Climate, 22, 499-517.

Liu, C., R. P. Allan, and G. J. Huffman, 2012: Co-variation of temperature and precipitation in CMIP5 models and satellite observations. Geophys. Res. Lett., 39, L13803, doi:10.1029/ 2012GL052093.

Lorenz, D. J., and E. T. DeWeaver, 2007: Tropopause height and zonal wind response to global warming in the IPCC scenario integrations. J. Geophys. Res., 112, D10119, doi:10.1029/2006JD008087.

Lu, J., G. Vecchi, and T. Reichler, 2007: Expansion of the Hadley cell under global warming. Geophys. Res. Lett., 34, L06805, doi:10.1029/2006GL028443.

Lyon, B., and D. G. DeWitt, 2012: A recent and abrupt decline in the East African long rains. Geophys. Res. Lett., 39, L02702, doi:10.1029/2011GL050337.

Meehl, G. A., and Coauthors, 2007: Global climate projections. Climate Change 2007: The Physical Science Basis, S. Solomon et al., Eds., Cambridge University Press, 747-845.

Mekis, E., and L. A. Vincent, 2011: An overview of the second generation adjusted daily precipitation dataset for trend analysis in Canada. Atmos.-Ocean, 49, 163-177.

Min, S., X. Zhang, and F. W. Zwiers, 2008: Human-induced Arctic moistening. Science, 320, 518-520.

Ming, Y., V. Ramaswamy, and G. Chen, 2011: A model investigation of aerosol-induced changes in boreal winter extratropical circulation. J. Climate, 24, 6077-6091.

Neelin, J. D., M. Munnich, H. Su, J. E. Meyerson, and C. E. Holloway, 2006: Tropical drying trends in global warming models and observations. Proc. Natl. Acad. Sci. USA, 103, 6110-6115.

Noake, K., D. Polson, G. C. Hegerl, and X. Zhang, 2012: Changes in seasonal land precipitation during the latter twentieth-century. Geophys. Res. Lett., 39, L03706, doi:10.1029/2011GL050405.

Randles, C., and V. Ramaswamy, 2008: Absorbing aerosols over Asia: A Geophysical Fluid Dynamics Laboratory general circulation model sensitivity study of model response to aerosol optical depth and aerosol absorption. J. Geophys. Res., 113, D21203, doi:10.1029/2008JD010140.

Santer, B. D., and Coauthors, 2007: Identification of humaninduced changes in atmospheric moisture content. Proc. Natl. Acad. Sci. USA, 104, 15244-15253.

Scheff, J., and D. M. W. Frierson, 2012: Robust future precipitation declines in CMIP5 largely reflect the poleward expansion of the model subtropical dry zones. Geophys. Res. Lett., 39, L18704, doi:10.1029/2012GL052910.

Schneider, U., A. Becker, P. Finger, A. Meyer-Christoffer, M. Ziese, and B. Rudolf, 2013: GPCC's new land-surface precipitation climatology based on quality-controlled in situ data and its role in quantifying the global water cycle. Theor. Appl. Climatol., doi:10.1007/s00704-013-0860-x, in press.

Seager, R., N. Naik, and G. Vecchi, 2010: Thermodynamic and dynamic mechanisms for large-scale changes in the hydrological cycle in response to global warming. J. Climate, 23, 4651-4668.

Seidel, D. J., Q. Fu, W. J. Randel, and T. J. Reichler, 2008: Widening of the tropical belt in a changing climate. Nat. Geosci., 1, $21-24$.

Smith, T. M., P. A. Arkin, M. R. P. Sapiano, and C. Chang, 2010: Merged statistical analyses of historical monthly precipitation anomalies beginning 1900. J. Climate, 23, 5755-5770.

Sutton, R. T., and B. Dong, 2012: Atlantic Ocean influence on a shift in European climate in the 1990s. Nat. Geosci., 5, 788792.

Taylor, K. E., R. J. Stouffer, and G. A. Meehl, 2012: An overview of CMIP5 and the experiment design. Bull. Amer. Meteor. Soc., 93, 485-498.

Vecchi, G. A., B. J. Soden, A. T. Wittenberg, I. M. Held, A. Leetmaa, and M. J. Harrison, 2006: Weakening of tropical Pacific atmospheric circulation due to anthropogenic forcing. Nature, 441, 73-76.

Von Storch, H., and F. W. Zwiers, 2001: Statistical Analysis in Climate Research. Cambridge University Press, 496 pp.

Vose, R. S., R. P. Schmoyer, P. M. Steurer, T. C. Peterson, R. Heim, T. R. Karl, and J. K. Eischeid, 1992: The Global Historical Climatology Network: Long-term monthly temperature, precipitation, sea level pressure, and station pressure data. Oak Ridge National Laboratory Rep. ORNL/CDIAC-53, NDP$041,324 \mathrm{pp}$

Wan, H., X. Zhang, F. W. Zwiers, and H. Shiogama, 2013: Effect of data coverage on the estimation of mean and variability of precipitation at global and regional scales. J. Geophys. Res., 118, 534-546, doi:10.1002/jgrd.50118.

Wang, G., E. A. B. Eltahir, J. A. Foley, D. Pollard, and S. Levis, 2004: Decadal variability of rainfall in the Sahel: Results from the coupled GENESIS-IBIS atmosphere-biosphere model. Climate Dyn., 22, 625-637.

Wentz, F. J., L. Ricciardulli, K. Hilburn, and C. Mears, 2007: How much more rain will global warming bring? Science, 317, 233 235

Willett, K. M., N. P. Gillett, P. D. Jones, and P. W. Thorne, 2007: Attribution of observed surface humidity changes to human influence. Nature, 449, 710-712.

Yin, J. H., 2005: A consistent poleward shift of the storm tracks in simulations of 21st century climate. Geophys. Res. Lett., 32, L18701, doi:10.1029/2005GL023684.

Yoshioka, W., N. M. Mahowald, A. J. Conley, W. D. Collins, D. W. Fillmore, C. S. Zender, and D. B. Coleman, 2007: Impact of desert dust radiative forcing on Sahel precipitation: Relative importance of dust compared to sea surface temperature variations, vegetation changes, and greenhouse gas warming. J. Climate, 20, 1445-1467.

Zhang, X., F. W. Zwiers, G. C. Hegerl, F. H. Lambert, N. P. Gillett, S. Solomon, P. A. Stott, and T. Nozawa, 2007: Detection of human influence on twentieth-century precipitation trends. Nature, 448, 461-465. 